\title{
Azetidines kill Mycobacterium tuberculosis without detectable resistance by blocking mycolate assembly.
}

\begin{abstract}
Alice Lanne, ${ }^{(a) \dagger}$ Yixin Cui, ${ }^{(b) \dagger}$ Edward Browne, ${ }^{(c)}$ Philip G. E. Craven, ${ }^{(b)}$ Nicholas J. Cundy, ${ }^{(b)}$ Nicholas J. Coltman, ${ }^{(d)}$ Katie Dale, ${ }^{(a)}$ Antonio Feula, ${ }^{(b)}$ Jon Frampton, ${ }^{(e)}$ Aaron Goff, ${ }^{(f)}$ Mariwan A. Hama Salih, ${ }^{(b)}$ Xingfen Lang,,$^{(g)}$ Xingjian Li, ${ }^{(b, g)}$ Christopher W. Moon, ${ }^{(b)}$ Michael Morton, ${ }^{(i)}$ Jordan Pascoe, ${ }^{(b)}$ Xudan Peng, ${ }^{(g)}$ Vanessa

Portman, ${ }^{(c)}$ Cara Press, ${ }^{(a)}$ Timothy Schulz-Utermoehl, ${ }^{(c)}$ Micky Tortorella, ${ }^{(g, j)}$ Zhengchao Tu, (g) Zoe E.

Underwood, ${ }^{(b)}$ Changwei Wang, ${ }^{(k)}$ Akina Yoshizawa, ${ }^{(b)}$ Tianyu Zhang,,$^{(k, l)}$ Simon J Waddell, ${ }^{(f) *}$ Joanna Bacon,,$^{(b)^{*}}$ Cleopatra Neagoie,,$^{(g, m)^{*}}$ John S. Fossey ${ }^{(b) \dot{\dagger}^{*}}$ and Luke J. Alderwick. ${ }^{(a))^{*}}$

(a) Institute of Microbiology and Infection, School of Biosciences, University of Birmingham, Edgbaston, Birmingham, West Midlands, B15

2TT, UK; (b) School of Chemistry, University of Birmingham, Edgbaston, Birmingham, West Midlands, B15 2TT, UK; (c) Sygnature

Discovery, The Discovery Building, BioCity, Pennyfoot Street, Nottingham, UK; (d) School of Biosciences, University of Birmingham, Edgbaston, Birmingham, West Midlands, B15 2TT, UK; (e) College of Medical and Dental Sciences, University of Birmingham, Edgbaston, Birmingham, West Midlands, B15 2TT, UK; (f) Department of Global Health and Infection, Brighton and Sussex Medical School, University of Sussex, Falmer, BN1 9PX, UK; (g) Guangzhou Institutes of Biomedicine and Health, Chinese Academy of Science, 190 Kai Yuan Avenue, Science Park, Guangzhou, China; (b) TB Research Group, National Infection Service, Public Health England, Manor Farm Road, Porton, Salisbury SP4 OJG, UK; (i) ApconiX Ltd, BIOHUB at Alderly Park, Nether Alderly, Cheshire, SK10 4TG, UK; (j) Legion Pharma, Kai Yuan Avenue, Science Park, Guangzbou, China; (k) State Key Laboratory of Respiratory Disease, Guangzhou Institutes of Biomedicine and Health (GIBH), Chinese Academy of Sciences (CAS), Guangzbou 510530, China; (l) University of Chinese Academy of Sciences (UCAS),

Beijing 100049, China;(m) Visiting Scientist, School of Chemistry, University of Birmingham, Edgbaston, Birmingham, West Midlands, B15 $2 T T, U K$.
\end{abstract}

t LJA and JSF share joint last authorship and along with CN, SJW and JB are co-corresponding authors with contact remit described later, YC and AL share joint first authorship. Remaining authors are listed alphabetically, and contributions for all authors are described in a later section.

\begin{abstract}
Tuberculosis (TB) is the leading cause of global morbidity and mortality resulting from infectious disease, with over 10 million new cases and 1.5 million deaths in 2019. This global emergency is exacerbated by the emergence of multi-drug-resistant MDR-TB and extensively-drug-resistant XDR-TB, therefore new drugs and new drug targets are urgently required. From a whole-cell phenotypic screen a series of azetidines derivatives termed $\mathrm{BGAz}$, that elicit potent bactericidal activity with $\mathrm{MIC}_{99}$ values $<10 \mu \mathrm{M}$ against drugsensitive Mycobacterium tuberculosis and MDR-TB were identified. These compounds demonstrate no detectable drug resistance. Mode of action and target deconvolution studies suggest that these compounds inhibit mycobacterial growth by interfering with cell envelope biogenesis, specifically late-stage mycolic acid biosynthesis. Transcriptomic analysis demonstrates that the BGAz compounds tested display a mode of action distinct from existing mycobacterial cell-wall inhibitors. In addition, the compounds tested exhibit toxicological and PK/PD profiles that pave the way for their development as anti-tubercular chemotherapies.
\end{abstract}

\section{Introduction}

Tuberculosis $(\mathrm{TB})$ is the principal infectious disease cause of death worldwide, accounting for 1.5 million deaths in 2019. One-third of the world's population is currently infected with latent TB, and over ten million new cases of active TB are recognised per annum. ${ }^{1,2}$ Patients suffering with TB are treated with a cocktail of 
four drugs over a six-month period. Whilst cure rates can be as high as $90-95 \%,{ }^{3}$ a combination of poor patient compliance and pharmacokinetic variability has led to the emergence of multi-drug-resistant (MDR) and extensively drug-resistant (XDR) TB. ${ }^{4,5}$ The alarming increase in MDR-TB (500,000 new cases in 2018), ${ }^{1}$ coupled with the fact that the last novel frontline anti-TB drug, Rifampicin, was discovered over 40 years ago $^{6}$ suggests development and implementation of new control measures are essential for the future abatement of $\mathrm{TB}^{7}$ Herein, the identity and anti-mycobacterial activity of azetidine derivatives with $\mathrm{MIC}_{99}$ values $<10 \mu \mathrm{M}$ against Mycobacterium tuberculosis are disclosed. These compounds did not give rise to emerging specific resistance in mycobacterial model organism Mycobacterium smegmatis and Mycobacterium bovis BCG. Mode of action and target deconvolution studies suggest mycobacterial growth inhibition is conferred by a hitherto uncharacterised mechanism that arrests late-stage mycolic acid biosynthesis. DMPK and toxicology profiles confirm that the azetidine derivatives identified display relevant and acceptable profiles for translation.

\section{Results}

\section{Identification and development of azetidine derivatives with anti-mycobacterial activity}

A bespoke compound library of novel lead-like small molecules ${ }^{8}$ for activity screening, that displayed a high fraction of $\mathrm{sp}^{3}\left(\mathrm{Fsp}^{3}\right.$, an indication of complexity and 3D-character) atoms, ${ }^{9-11}$ that were free from pan-assay interference compounds (PAINS) $)^{12,13}$ and are synthetically tractable allowing for hit-to-lead scaffold elaboration ${ }^{14}$ were sought. Unrelated synthetic chemistry methodology studies ${ }^{15}$ proved to be an ideal untapped source of such compounds. ${ }^{16-19}$ Compounds were fed into an open-ended anti-mycobacterial compound screen at the University of Birmingham Drug Discovery Facility. ${ }^{20}$ From which, an azetidine derivative BGAz-001 (general formula Figure 1: $\mathrm{R}^{1}=\mathrm{R}^{2}=\mathrm{H}, \mathrm{R}^{3}=\mathrm{Br}, \mathrm{R}^{4}$ and $\mathrm{R}^{5}=$ pyrrolidine fragment) that displayed promising anti-mycobacterial activity against both Mycobacterium smegmatis and Mycobacterium bovis BCG, with MICs of $30.5 \mu \mathrm{M}$ and $64.5 \mu \mathrm{M}$, respectively (Table 1, entry 1) was identified and further azetidine derivatives were synthesised at Guangzhou Institutes of Biomedical Health $(\mathrm{GIBH}){ }^{21}$ Analysis of calculated medicinal chemistry properties and appraisal of preliminary screening against BCG at $2 \mu \mathrm{M}$ and $20 \mu \mathrm{M}$ in an end point REMA assay for anti-mycobacterial activity, alongside secondary MIC determination against BCG (MIC refers to $\mathrm{MIC}_{99}$ unless otherwise stated), was sufficient to rule out the majority of ancillary compounds for further study. ${ }^{22}$ Thus four additional azetidine-analogues with good activity against model organisms, were retained for further evaluation of anti-TB activity (Figure 1 and Table 1, entries 2-5: BGAz-002: $\mathrm{R}^{1}=\mathrm{OCF}_{3}$, $\mathrm{R}^{2}=\mathrm{H}, \mathrm{R}^{3}=\mathrm{OCF}_{3}$ and $\mathrm{R}^{4} \& \mathrm{R}^{5}=$ pyrrolidine fragment; BGAz-003: $\mathrm{R}^{1}=\mathrm{H}, \mathrm{R}^{2}=\mathrm{CF}_{3}, \mathrm{R}^{3}=\mathrm{Br}$ and $\mathrm{R}^{4}$ \& $\mathrm{R}^{5}=$ pyrrolidine fragment; BGAz-004: $\mathrm{R}^{1}=\mathrm{H}, \mathrm{R}^{2}=\mathrm{Br}, \mathrm{R}^{3}=\mathrm{Br}$ and $\mathrm{R}^{4} \& \mathrm{R}^{5}=$ pyrrolidine fragment; and BGAz-005 where $\mathrm{R}^{1}=\mathrm{OCF}_{3}, \mathrm{R}^{2}=\mathrm{H}, \mathrm{R}^{3}=\mathrm{OCF}_{3}, \mathrm{R}^{4}=$ methyl and $\mathrm{R}^{5}=\mathrm{H}$, Table 1, entries 2-5) 


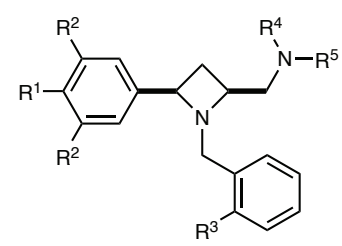

\begin{tabular}{llllll} 
(rac) & $\mathrm{R}^{1}$ & $\mathrm{R}^{2}$ & $\mathrm{R}^{3}$ & $\mathrm{R}^{4}$ & $\mathrm{R}^{5}$ \\
\hline BGAz-001 & $\mathrm{H}$ & $\mathrm{H}$ & $\mathrm{Br}$ & \\
BGAz-002 & $\mathrm{OCF}_{3}$ & $\mathrm{H}$ & $\mathrm{OCF}_{3}$ & - \\
BGAz-003 & $\mathrm{H}$ & $\mathrm{CF}_{3}$ & $\mathrm{Br}$ & $\mathrm{N}$ \\
BGAz-004 & $\mathrm{H}$ & $\mathrm{Br}$ & $\mathrm{Br}$ & $-\mathrm{OCF}_{3}$ \\
BGAz-005 & $\mathrm{OCF}_{3}$ & $\mathrm{H}$ & $\mathrm{OCF}_{3}$ & $\mathrm{H}$ & $\mathrm{CH}_{3}$ \\
\hline
\end{tabular}

Figure 1. University of Birmingham and Guangzhou Institutes of Biomedicine and Health co-developed Azetidines (BGAz) with antitubercular activity.

Anti-tubercular activity of azetidine derivatives (BGA₹-001-BGA₹-005)

Compounds BGAz002-BGAz005 displayed antitubercular activity against $M$. tuberculosis strains that include reference strains H37Ra::pTYOK and H37Rv, and two clinical isolates $M$. tuberculosis (Beijing/W lineage 1192/015) and M. tuberculosis (Beijing 08/00483E) that are drug-sensitive or multi-drug-resistant to isoniazid, rifampicin, pyrazinamide and ethambutol (Table 1). 
Table 1. MIC and MBC values of the BGAz-001-BGAz-005 against mycobacterial strains with different drug-susceptibility profiles. The $M$. tb H37Ra::pTYOK is an autoluminescent strain of mycobacteria. ${ }^{23}$ The MIC99 values of BGAz-001-BGAz-005 against M. smegmatis and M. bovis BCG, drug-sensitive M. tuberculosis $\mathrm{H} 37 \mathrm{Rv}$ (reference strain) and M. tuberculosis 1192/015 (clinical isolate), and multi-drug-resistant $M$. tuberculosis 08/00483E (clinical isolate resistant to INH, RIF, PZA and EMB). MIC values were determined from three biological replicates using a resazurin endpoint assay. The minimum bactericidal concentration (MBC) was determined for BGAz-002-BGAz-005 against $M$. bovis BCG. NT = not tested.

\begin{tabular}{|c|c|c|c|c|c|c|c|c|}
\hline Entry & Compound & $\begin{array}{l}\text { M. smeg. } \\
\text { MIC }_{99} \\
(\mu \mathrm{M})^{(a)}\end{array}$ & $\begin{array}{l}\text { BCG } \\
\text { MIC } 99 \\
(\mu M)^{(a)}\end{array}$ & $\begin{array}{l}\text { BCG } \\
\text { MBC } \\
(\mu \mathrm{M})\end{array}$ & $\begin{array}{l}\text { M. } t b \\
\text { H37Ra::pTY } \\
\text { OK MIC } \\
(\mu \mathrm{M})\end{array}$ & $\begin{array}{l}\text { M. } t b \\
\text { H37Rv } \\
\text { MIC } \\
(\mu \mathrm{M})^{(a)} \\
\end{array}$ & $\begin{array}{l}\text { M. } t b \\
1192 / 015 \\
\text { MIC }_{99} \\
(\mu \mathrm{M})^{(a)} \\
\end{array}$ & $\begin{array}{l}M D R-M . t b \\
08 / 00483 \mathrm{E} \\
\text { MIC }_{99} \\
(\mu \mathrm{M})^{\text {(a) }} \\
\end{array}$ \\
\hline 1 & & 30.5 & 65.0 & NT & $>30$ & NT & NT & NT \\
\hline 2 & & 32 & 15 & $12-25$ & 9.2 & 6.2 & 37.8 & 33.5 \\
\hline 3 & & 26 & 25 & $12-25$ & 6.9 & 3.3 & 19.1 & 16.8 \\
\hline 4 & & 14 & 14 & $12-25$ & 7.03 & 3.3 & 15.4 & 15.6 \\
\hline 5 & & 24 & 21 & $12-25$ & 4.5 & 7.2 & 23.2 & 2.8 \\
\hline
\end{tabular}

Compounds BGAz-002-BGAz-005 elicited anti-tubercular activities ranging from 4.5-9.2 $\mu \mathrm{M} \mathrm{MIC}_{\text {lux } 50}$ using a recently reported autoluminescent avirulent strain of $M$. tuberculosis H37Ra (Table 1, entries 2-5). Both BGAz-003 and BGAz-004 inhibit M. tuberculosis (H37Rv) at an MIC of $3.3 \mu \mathrm{M}$ (Table 1, entries 3 and 4), with BGAz-002 and BGAz-005 inhibiting growth at MICs of $6.2 \mu \mathrm{M}$ and $7.2 \mu \mathrm{M}$, respectively (Table 1, entries 2 and 5). The MICs determined for BGAz-002-BGAz-005 are higher in the drug-sensitive $M$. tuberculosis Beijing/W (1192/015) clinical isolate compared to H37Rv. When comparing MICs between drugsensitive and drug MDR clinical strains, no significant differences were observed for BGAz-002-BGAz004, suggesting that the acquisition of mutations conferring front-line drug resistance does not impact upon the antitubercular activity of these compounds. However, it is noteworthy that when BGAz-005 was tested against $M$. tuberculosis 08/00483E (a clinical isolate that is resistant to isoniazid, rifampicin, pyrazinamide and ethambutol), ${ }^{24}$ this compound displayed an eight-fold lower MIC (2.8 $\mu \mathrm{M}$, Table 1, entry 5$)$ in comparison to the drug-sensitive isolate. The absence of cross-resistance of these new anti-TB agents with the current 
frontline TB drugs is an important consideration in development of new TB therapies with distinct modes of action. ${ }^{25}$ Evaluation of the Minimal Bactericidal Concentrations (MBCs) for BGAz-002-BGAz-005 against BCG demonstrates that these compounds exhibit bactericidal activity, since both MIC and MBC values overlap (Table 1).

Physiochemical and Toxicological properties of $B G A z-002-B G A z-005$

Table 2. Physiochemical and toxicological properties of BGAz-002-BGAz-005.

\begin{tabular}{|c|c|c|c|c|c|c|c|}
\hline Entry & Compound & $\begin{array}{l}\begin{array}{l}\text { Kinetic } \\
\text { solubility } \\
(\mu \mathrm{M})^{(a)}\end{array} \\
\end{array}$ & $\begin{array}{l}\text { Mouse } \\
\text { PPB } \\
(\%)(b)\end{array}$ & $\begin{array}{l}\text { Mouse Microsomal } \\
\text { CL }_{\text {int }} \text { clearance } \\
(\mu \mathrm{L} / \mathrm{min} / \mathrm{mg})^{(c)}\end{array}$ & $\begin{array}{l}\text { Mouse Hepatocyte } \\
\mathrm{CL}_{\text {int }} \text { clearance } \\
(\mu \mathrm{L} / \mathrm{min} / \mathrm{mg})^{(d)}\end{array}$ & $\begin{array}{l}\text { Caco-2 B- } \\
\text { A Paap } \\
10^{6} \mathrm{~cm} / \mathrm{s}(e)\end{array}$ & $\begin{array}{l}\text { Caco-2 } \\
\text { Efflux } \\
\text { ratio (e) }\end{array}$ \\
\hline 1 & & 57 & $>99$ & 166 & 60 & 2.8 & 1.2 \\
\hline 2 & & 27 & $>99$ & 179 & 52 & 1.1 & 3.6 \\
\hline 3 & & 9 & $>99$ & 192 & 35 & 0.5 & 1.4 \\
\hline 4 & & 117 & $>99$ & 36 & 37 & 1.4 & 0.9 \\
\hline
\end{tabular}

BGAz-002-BGAz-005 were subjected to in vitro DMPK testing; BGAz-001 was excluded from further study due to its comparatively poor anti-mycobacterial activity. The poorer kinetic solubility BGAz-002-BGAz$\mathbf{0 0 4}(9$ to $57 \mu \mathrm{M})$ in aqueous buffered solution in comparison to the higher solubility of BGAz-005 $(117 \mu \mathrm{M})$ can be attributed to the presence of a secondary versus tertiary amine functionality (Table 2). Metabolic stability of the compounds was evaluated through measuring the intrinsic clearance (CLint) by mouse liver microsomes and by liver hepatocytes. Compounds BGAz-002-BGAz004 all exhibited a CL int. $_{\text {of }}>150$ $\mu \mathrm{L} / \mathrm{min} / \mathrm{mg}$ in the microsomal stability assay indicating a rapid clearance (Table 2, entries 1 to 3), with BGAz-005 giving the lowest rate of microsomal clearance (36 $\mu \mathrm{L} / \mathrm{min} / \mathrm{mg}$, Table 2, entry 4). Experiments were repeated using mouse liver hepatocytes and all four compounds afforded CL $_{\text {int }}$ values of $<60$ $\mu \mathrm{L} / \mathrm{min} / \mathrm{mg}$, indicating good overall metabolic stability (Table 2). Caco-2 permeability assays were conducted to predict both intestinal permeability and drug efflux. Compounds BGA-002-BGAz-004 exhibited poor efflux ratios whilst BGAz-005 continued to perform well with an efflux ratio of less than 1.0. 
The pharmacokinetic (PK) parameters of BGAz-001-BGAz-005 in a mouse model were investigated by cassette (combined) dosing at $5 \mathrm{mg} / \mathrm{kg}$ PO, $1 \mathrm{mg} / \mathrm{kg}$ IV and IP (Supplementary PK Data file: Tables 1 to 5). For the oral dosing, at $5 \mathrm{mg} / \mathrm{kg}, \mathbf{B G A z - 0 0 1 , ~ B G A z - 0 0 2}$ and BGAz-003 gave peak serum concentrations $\left(\mathrm{C}_{\max }\right)$ of 54.7, 53.9 and $56.0 \mu \mathrm{g} / \mathrm{L}$ respectively. BGAz-004 gave the highest $\mathrm{C}_{\max }$ whereas BGAz-005 gave the lowest, 87.6 and $43.1 \mu \mathrm{g} / \mathrm{L}$ respectively (Table 3, entries 2 and 3). The plasma half-lives $\left(T_{1 / 2}\right.$ ) revealed BGAz001 to be 1.5 h, BGAz002 and BGAz003 to be 24.9 and 28.0 h respectively. BGAz004 has a half-life of $11.4 \mathrm{~h}$ with BGAz005 having the longest half-life of 35.7 h. BGAz001-003 were excluded from further study due to poor PK/PD parameters. Mice were dosed multiple times with BGAz-004 and BGAz-005 (four and three times respectively) at $30 \mathrm{mg} / \mathrm{kg}$ (PO) in order to provide evidence of compound tolerability and refinement of measured parameters. BGAz-004 and BGAz-005 gave $\mathrm{C}_{\max }$ values of 363.0 and 1712.5 $\mu \mathrm{g} / \mathrm{L}$ (respectively); $T_{1 / 2}$ values of 8.1 and 80.4 hours were calculated respectively (Table 3, entries 2 and 3). The total body exposure from multiple dosing at $30 \mathrm{mg} / \mathrm{kg}$ (PO) of BGAz-005 (82247.7 ng/mL*h) was significantly greater than BGAz-004 (5420.99 $\mathrm{ng} / \mathrm{mL}^{*} \mathrm{~h}$ ), indicating a superior overall pharmacokinetic profile for BGAz-005 (Table 3, entries 2 and 3). BGAz-002, BGAz-004 and BGAz-005 were tested for cytochrome P450 (CYP450) metabolic activity by measuring the inhibition each of specific enzymes in human liver microsomes. All three compounds exhibited no discernible inhibition of CYP1A2, an enzyme known to metabolise aromatic/heterocyclic amine-containing drugs (Table 3). The CYP2C9 enzyme is a relatively abundant CYP450 in the liver that dominates CYP450-mediated drug oxidation. In this regard, only minimal CYP2C9 inhibition when BGAz-004 was pre-incubated for $30 \mathrm{~min}$ prior to the addition of NADPH to initiate catalysis was observed. Known to metabolise a wide range of drug molecules, CYP2C19 is an essential member of the CYP450 superfamily as it contributes $\sim 16 \%$ of total hepatic content in humans. While BGAz-002 and BGAz-005 displayed only negligible inhibition of this enzyme, BGAz-004 displays strong inhibitory activity when pre-incubated for $30 \mathrm{~min}$ prior to initiation of catalysis (Table 3). CYP2D6 is widely implicated in the metabolism of drugs that contain amine functional groups, such as monoamine oxidase inhibitors and serotonin reuptake inhibitors. CYP2D6 is responsible for the second highest number of drugs metabolised by the CYP450s, as demonstrated by the significant inhibition of this enzyme by BGAz002, BGAz-004 and BGAz-005. All four compounds were evaluated for mitochondrial dysfunction by measuring $\mathrm{IC}_{50}$ values against $\mathrm{HepG} 2$ cells cultured in media containing either glucose or galactose, which serves to direct cellular metabolic activity towards glycolysis or oxidative phosphorylation, respectively. Whilst BGAz-004 exhibited a negligible effect both BGAz-002 and BGAz-005 demonstrated cytotoxicity with respective $\mathrm{IC}_{50}$ values of $38 \mu \mathrm{M}$ and $21 \mu \mathrm{M}$, respectively (Table 3). In order to enhance cellular susceptibility to mitochondrial toxicants, assays were repeated in the presence of galactose which resulted in Glu/Gal ratios of $<1$, confirming no mitochondrial toxicity. Compounds BGAz-002, BGAz-004 and 
BGAz-005 were assayed for hERG inhibition using IonWorks patch clamp electrophysiology. An eight-point concentration response curve was generated from three-fold serial dilution of a top compound concentration of $167 \mu \mathrm{M}$. Compound BGAz-002 was the best-performing compound, displaying a hERG liability $\mathrm{IC}_{50}$ of $173 \mu \mathrm{M}$, (i.e. inhibition of less than $50 \%$ at top $167 \mu \mathrm{M}$ test concentration). In comparison, compounds BGAz-004 and BGAz-005 performed significantly worse with hERG liability $\mathrm{IC}_{50}$ of $25 \mu \mathrm{M}$ and $12.7 \mu \mathrm{M}$, respectively. Overall, the BGAz compounds investigated in this study display an encouraging toxicological and $\mathrm{PK} / \mathrm{PD}$ profile to enable further exploration and development towards the clinic. 
Table 3. Pharmacokinetic profiles, CYP450 activities, mitochondrial dysfunction and hERG liabilities of BGAz-002, BGAz-004 and BGAz-005.

\begin{tabular}{|c|c|c|c|c|c|c|c|c|c|c|c|c|}
\hline Entry & Compound & $\begin{array}{l}\text { Dose } \\
(\mathrm{mg} / \mathrm{kg})\end{array}$ & $\begin{array}{l}\mathrm{C}_{\max } \\
\text { (Mouse, P.O.) } \\
(\mu \mathrm{g} / \mathrm{L})^{(a)}\end{array}$ & $\begin{array}{l}T_{\max } \\
\text { (Mouse, P.O.) } \\
\text { (h) (b) }\end{array}$ & $\begin{array}{l}\mathrm{T}_{1 / 2} \\
\text { (Mouse, P.O.) } \\
\text { (h) (c) }\end{array}$ & $\begin{array}{l}\mathbf{A U C}_{0 \text {-inf }} \\
\left(\mathrm{ng} / \mathrm{mL}^{*} \mathbf{h}\right)^{(d)}\end{array}$ & $\begin{array}{l}\text { CYP1A2 } \\
\text { [pre-inc] } \\
\text { IC }_{50}(\mu \mathbf{M})\end{array}$ & $\begin{array}{l}\text { CYP2C9 } \\
\text { [pre-inc] } \\
\text { IC }_{50}(\mu \mathbf{M})\end{array}$ & $\begin{array}{l}\text { CYP2C19 } \\
\text { [pre-inc] } \\
\text { IC }_{50}(\mu \mathrm{M})\end{array}$ & $\begin{array}{l}\text { CYP2D6 } \\
\text { [pre-inc] } \\
\text { IC }_{50}(\mu \mathrm{M})\end{array}$ & $\begin{array}{l}\text { HepG2 } \\
\text { Cytotoxicity } \\
\text { [Glu/Gal] } \\
\text { IC }_{50}(\mu \mathrm{M}) \\
\end{array}$ & $\begin{array}{l}\text { hERG } \\
\text { IC } \\
(\mu \mathrm{M})\end{array}$ \\
\hline 1 & & $5^{(e)}$ & 53.9 & 1.87 & 24.9 & 1268.03 & $>20[>20]$ & $>20[>20]$ & $>20[>20]$ & $0.19[0.08]$ & $38 / 65$ & 173 \\
\hline \multirow[t]{2}{*}{2} & & & & & & & $>20[>20]$ & $>20[17.78]$ & $>15.13[2.04]$ & $1.97[1.05]$ & $>100 />100$ & 25 \\
\hline & $(r a c) \mathrm{B}$ & $30 \oplus$ & 363 & 1.0 & 8.07 & 5420.99 & & & & & & \\
\hline \multirow{3}{*}{3} & & $5^{(e)}$ & 43.1 & 6 & 35.7 & 1542.4 & & & & & & \\
\hline & & & & & & & $>20[>20]$ & $>20[>20]$ & $>20[15.79]$ & $0.06[0.01]$ & $21 / 23$ & 12.7 \\
\hline & (rac) BGAZ-005 & $30(2)$ & 1712.54 & 0.87 & 80.4 & 82247.7 & & & & & & \\
\hline
\end{tabular}




\section{BGAz compounds kill M. tuberculosis with bactericidal activity}

The bactericidal activity of BGAz-004 and BGAz-005 against $M$. tuberculosis H37Rv was further assessed by exposing the bacilli to a range of concentrations of BGAz004 and BGAz-005 over a time-course of 14 days and total viable counts $\left(\mathrm{CFUmL}^{-1}\right)$ enumerated on solid medium. Both BGAz-004 and BGAz-005 were active against $M$. tuberculosis H37Rv (Figure 2, panels A and B) with BGAz-005 demonstrating statistically significantly greater early bactericidal and concentration-dependent activity than BGAz-004 at day six $(P=$ 0.046) and day ten $(P=0.049)$ (Figure 2D). Exposure of $M$. tuberculosis H37Rv to BGAz-005 resulted in a greater bactericidal effect with more pronounced activity earlier in the time-course, with a reduction of 3.28 $\pm 1.00 \log _{10} \mathrm{CFUmL}^{-1}$ after six days of exposure and reduction of $3.99+0.60 \log _{10} \mathrm{CFUmL}^{-1}$ after 14 days' exposure, at a concentration of $96 \mu \mathrm{M}$ (Figure 2B). Equivalent activity was not observed by BGAz-004 early in the time-course and showed delayed activity at all concentrations, only achieving a decrease in $0.79 \pm 2.20$ $\log _{10} \mathrm{CFUmL}^{-1}$ by day six and $1.90 \pm 1.28 \log _{10} \mathrm{CFUmL}^{-1}$ reduction after 14 days at $96 \mu \mathrm{M}$ (Figure 2A). The profile for $\mathbf{B G A}_{\mathbf{z}} \mathbf{- 0 0 4}$ is commensurate with antibiotics that exhibit bacteriostatic activity at lower concentrations. Isoniazid, demonstrated a higher rate of bactericidal activity compared to both BGAz-004 and BGAz-005 by achieving a reduction of $>4.52 \pm 0.60 \log _{10} \mathrm{CFUmL}^{-1}$, to a limit of detection $\left(100 \mathrm{CFUmL}^{-}\right.$ ${ }^{1}$ ), by day ten, at a lower concentration of $29 \mu \mathrm{M}$ (Figure 2C). 

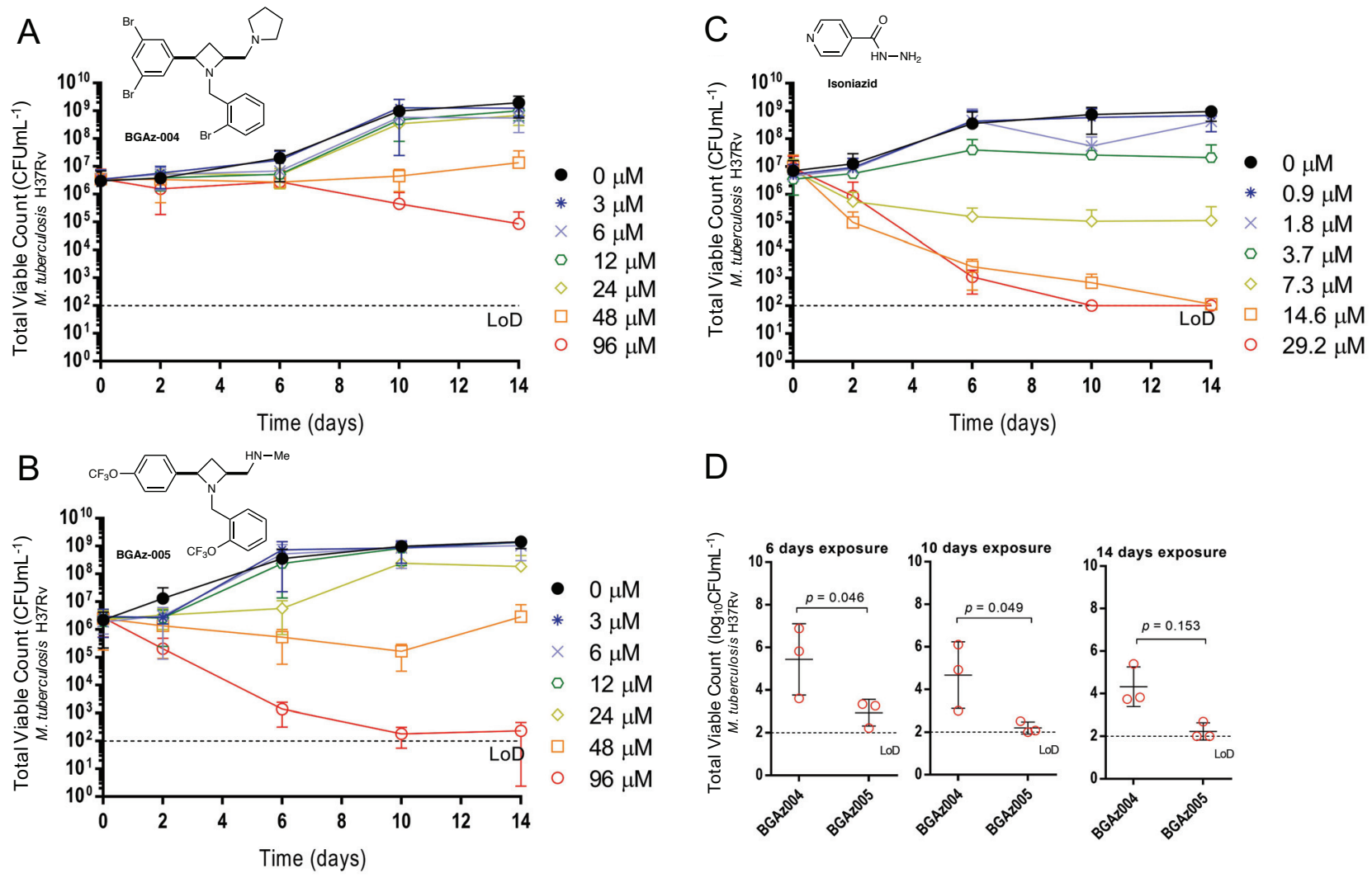

Figure 2. Assessment of bactericidal activity of BGAz-004 and BGAz-005 against $M$. tuberculosis H37Rv. Average total viable counts $\left(\mathrm{CFUmL}{ }^{-1}\right)$ of $M$. tuberculosis cultures exposed to either BGAz-004 (Panel A) or BGAz-005 (Panel B) at concentrations: $0 \mu \mathrm{M}$ (0.1\% DMSO) (circle, closed), $3 \mu \mathrm{M}, 6 \mu \mathrm{M}, 12 \mu \mathrm{M}, 24 \mu \mathrm{M}, 48$ $\mu \mathrm{M}$ and $96 \mu \mathrm{M}$, or isoniazid (Panel C) at concentrations $0 \mu \mathrm{M}(0.1 \% \mathrm{DMSO}), 0.9 \mu \mathrm{M}, 1.8 \mu \mathrm{M}, 3.7 \mu \mathrm{M}, 7.3 \mu \mathrm{M}, 14.6 \mu \mathrm{M}$ and $29.2 \mu \mathrm{M}$ over a 14 -day time-course. Samples were taken after 0,2, 6, 10, and 14 days of antibiotic exposure, serially diluted, and plated by the method of Miles et al.26 Statistical comparisons were performed at 6,10 and 14 days of antibiotic exposure at $96 \mu \mathrm{M}$ BGAz-004 and BGAz-005 using factorial ANOVA and post-hoc Tukey's honestly significant difference test (Panel D). Data represents three biological repeats \pm standard deviation.

In addition to the enumeration of viable bacilli on agar, the activity of BGAz-004 and BGAz-005 was determined using flow cytometry. This approach allows for a direct assessment of whether BGAz compounds are able to kill M. tuberculosis in a dose dependent manner and whether the killing profile was similar between these compounds and to that observed for isoniazid, which would provide insights about their mode of action. ${ }^{27}$ Culture samples were taken at each time-point and dual-stained using Calcein Violet with an acetoxy-methyl ester group (CV-AM) that is a correlate of metabolic activity, and Sytox Green (SG) that enables measurement of cell-wall permeability (a proxy for cell death). Single bacilli were identified by forward scattered light area and height using flow cytometry analyses. Gated single cells were further differentiated based upon the presence and absence of CV-AM and SG staining using a quadrant gating approach. The percentages of the population that are unstained or stained with each dye (or both dyes) is represented in four gates P1-P4 (P1: CV-AM-/SG-, P2: CV-AM ${ }^{+} / \mathrm{SG}^{-}, \mathrm{P} 3: \mathrm{CV}-\mathrm{AM}^{+} / \mathrm{SG}^{+}, \mathrm{P} 4: \mathrm{CV}_{-} \mathrm{AM}^{-}$ $/ \mathrm{SG}^{+}$) (Figure 3). The CV-AM staining profiles (metabolic activity) for these compounds were reflective of the total viable counts (Figure 3); the decrease in CV-AM staining over the time-course at $96 \mu \mathrm{M}$ for BGAz- 
005 was statistically significant after day six $(P=0.043)$, day ten $(P=0.08)$, and day fourteen $(P=0.011)$ compared to the decrease in the CV-AM staining for BGAz-004, at the same concentration (Figure 3A and B; P2). A similar difference in activity was observed at $48 \mu \mathrm{M},(P=0.067,0.065,0.066$ for days 6,10 and 14 , respectively). The SG-staining profiles showed that both compounds possessed equivalent killing activity at high concentrations of $96 \mu \mathrm{M}$ (Figure 3A and B; P4); however, BGAz-005 shows higher levels of kill at days six and fourteen with a lower concentration of $48 \mu \mathrm{M}(P=0.027$ and 0.068 , respectively). Both BGAz-004 and BGAz-005 show a similar staining profiles to isoniazid (Figure 3C), which targets the mycobacterial cellwall. ${ }^{27}$

A

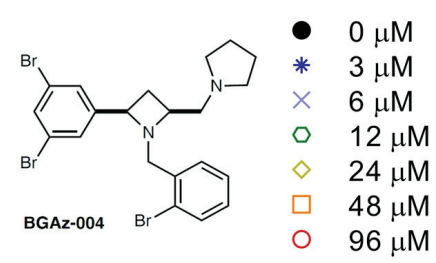

P1

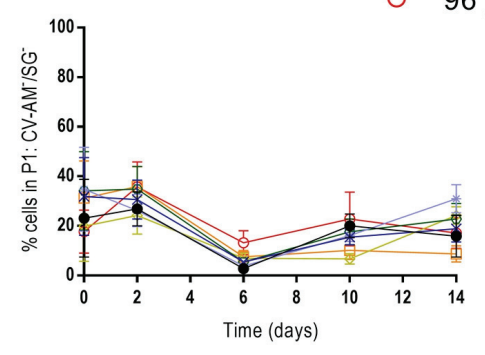

P2

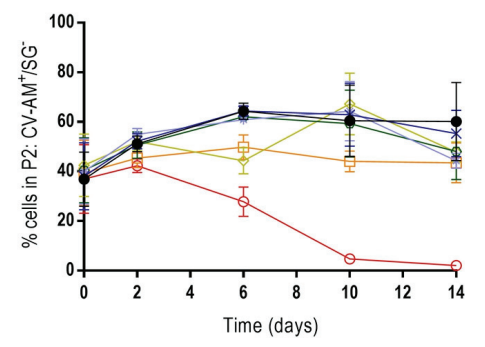

P3

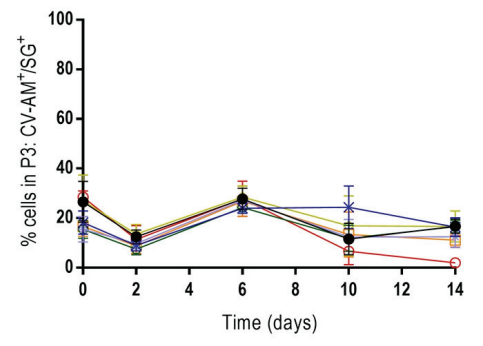

P4

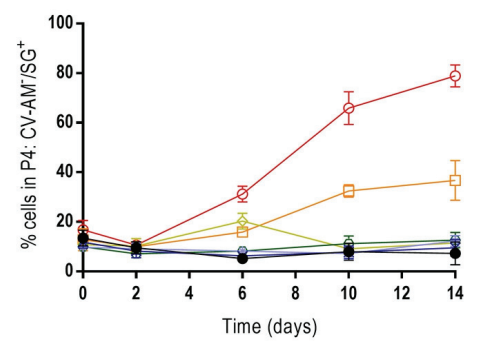

\section{B}
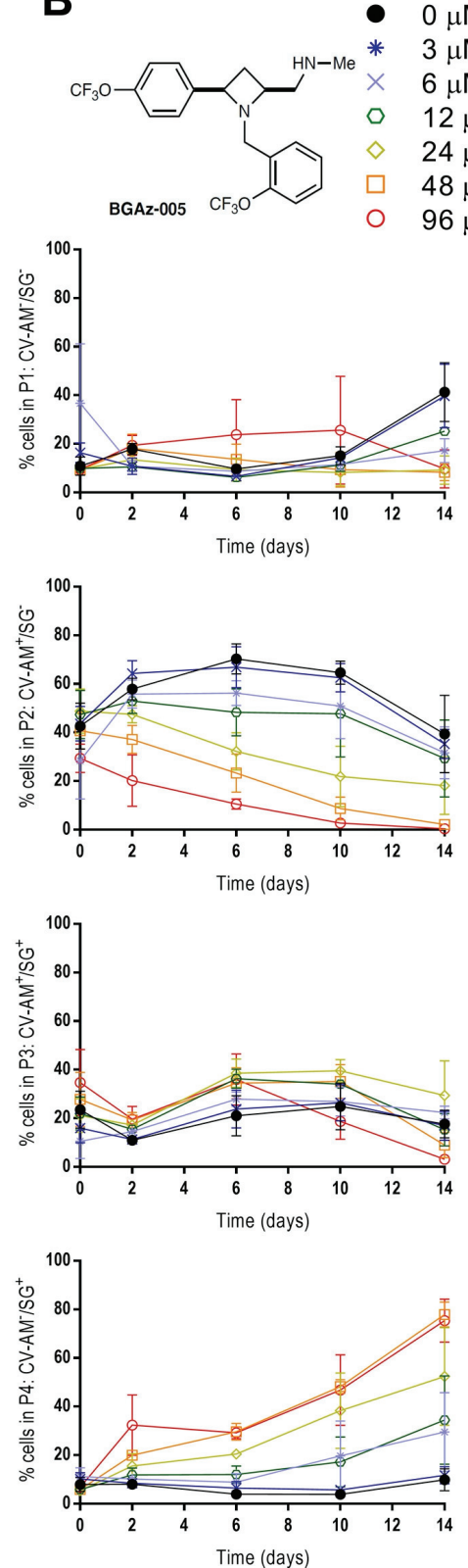
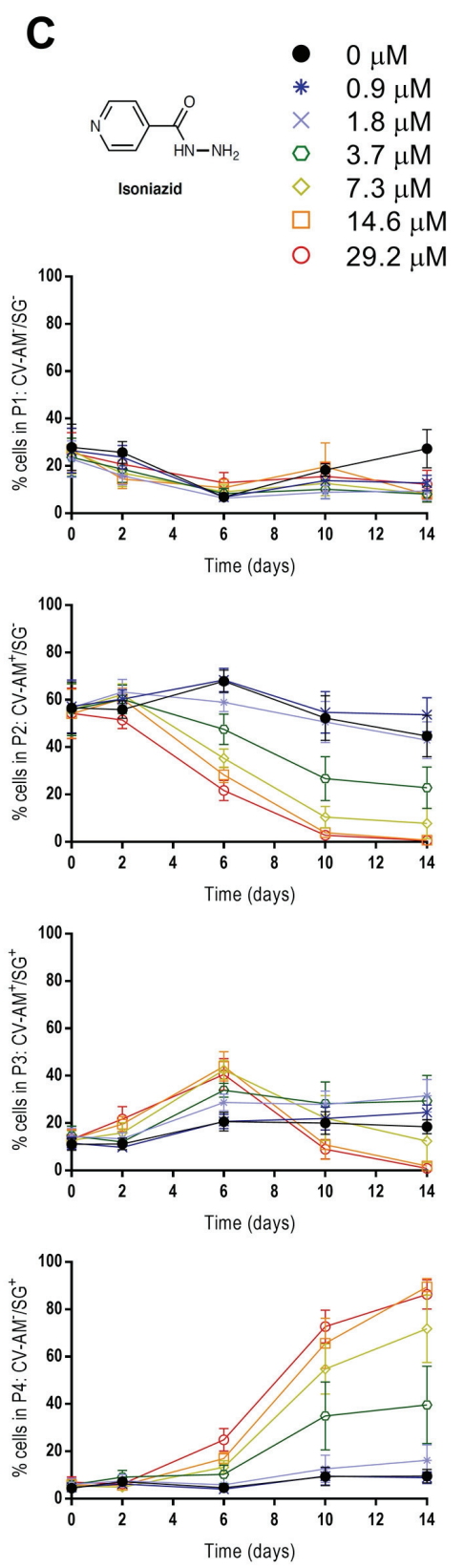

Figure 3. Assessment of bactericidal activity of BGAz-004 and BGAz-005 against $M$. tuberculosis H37Rv. Quantitation of Calcien-Violet-AM (CV-AM) and Sytox-green (SG) fluorescence of M. tuberculosis H37Rv, using flow cytometry, after exposure to BGAz-004 (column A), BGAz-005 (column B) at 
concentrations: $0 \mu \mathrm{M}(0.1 \%$ DMSO), $3 \mu \mathrm{M}, 6 \mu \mathrm{M}, 12 \mu \mathrm{M}, 24 \mu \mathrm{M}, 48 \mu \mathrm{M}$ and $96 \mu \mathrm{M}$ or (column C) isoniazid at concentrations $0 \mu \mathrm{M}(0.1 \% \mathrm{DMSO}), 0.9 \mu \mathrm{M}, 1.8$ $\mu \mathrm{M}, 3.7 \mu \mathrm{M}, 7.3 \mu \mathrm{M}, 14.6 \mu \mathrm{M}$ and $29.2 \mu \mathrm{M}$ over a 14-day time-course. The percentages of the population that are unstained or stained with each dye (or both dyes) is represented in four gates (rows P1-P4). Row P1: unstained population (CV-AM-/SG-); row P2: CV-stained population (CV-AM+/SG-); row P3: dualstained population $\left(\mathrm{CV}-\mathrm{AM}^{+} / \mathrm{SG}^{+}\right)$; and row P4: SG-stained population $\left(\mathrm{CV}-\mathrm{AM}^{-} / \mathrm{SG}^{+}\right)$. Data represents three biological repeats \pm standard deviation. Statistical comparisons were made using factorial ANOVA and post-hoc Tukey's honestly significant difference test.

$B G A z_{-} 004$ and BGAz-005 inhibit the incorporation of mycobacterial cell-wall precursors and display no detectable resistance.

Whole genome sequencing (WGS) of laboratory-generated mutants that are resistant to TB drugs is a widely used approach to determine the mode of action of novel antibacterial compounds. ${ }^{28-30}$ Multiple attempts $(>5$ biological repeats) to generate drug-resistant mutants of BGAz-002-BGAz-005 in M. smegmatis and M. bovis BCG (including a strain of BCG devoid of $r e c G$ which has a higher mutational frequency) ${ }^{31}$ were unsuccessful, implying an undetectably low frequency of resistance for these compounds (Supplementary Information). This advantageous property is a double-edged sword. The discovery of the BGAz series as novel antitubercular compounds with low frequencies of resistance is attractive in terms of drug development, especially in the context of MDR-TB; however, the inability to generate resistant mutants against the most active compounds suggests that this series of compounds may elicit pleiotropic activity or have non-specific modes of action, or non-protein target(s). Therefore, in order to investigate the mode of action of BGAz005, the most active of the compounds tested against mycobacteria, biosynthetic inhibition of five major macromolecular pathways were evaluated by measuring incorporation of selected radiolabelled precursors during microbial cell culture. Addition of BGAz-005 up to a concentration of $0.75 \times$ MIC had almost no effect of the incorporation of $\left[{ }^{3} \mathrm{H}\right]$-thymidine, $\left[{ }^{3} \mathrm{H}\right]$-uridine and $\left[{ }^{3} \mathrm{H}\right]$-leucine with only a moderate $20 \%$ reduction of incorporation at $1 \times$ MIC, suggesting that BGAz-005 does not directly inhibit DNA, RNA or protein biosynthesis (Figure 4). In contrast, BGAz-005 decreased the incorporation of both $\left[{ }^{3} \mathrm{H}\right]-\mathrm{DAP}$ and $\left[{ }^{14} \mathrm{C}\right]$-acetic acid from six hours post-labelling and at $0.5 \times$ and $1 \times \mathrm{MIC}$, caused a titratable decrease in $\left[{ }^{14} \mathrm{C}\right]-$ acetic acid incorporation, exerting a $\sim 50 \%$ and $\sim 75 \%$ loss of lipid biosynthesis, respectively (Figure 4 ). These data suggest that the BGAz-005 acts by inhibiting aspects of mycobacterial cell envelope biosynthesis. 

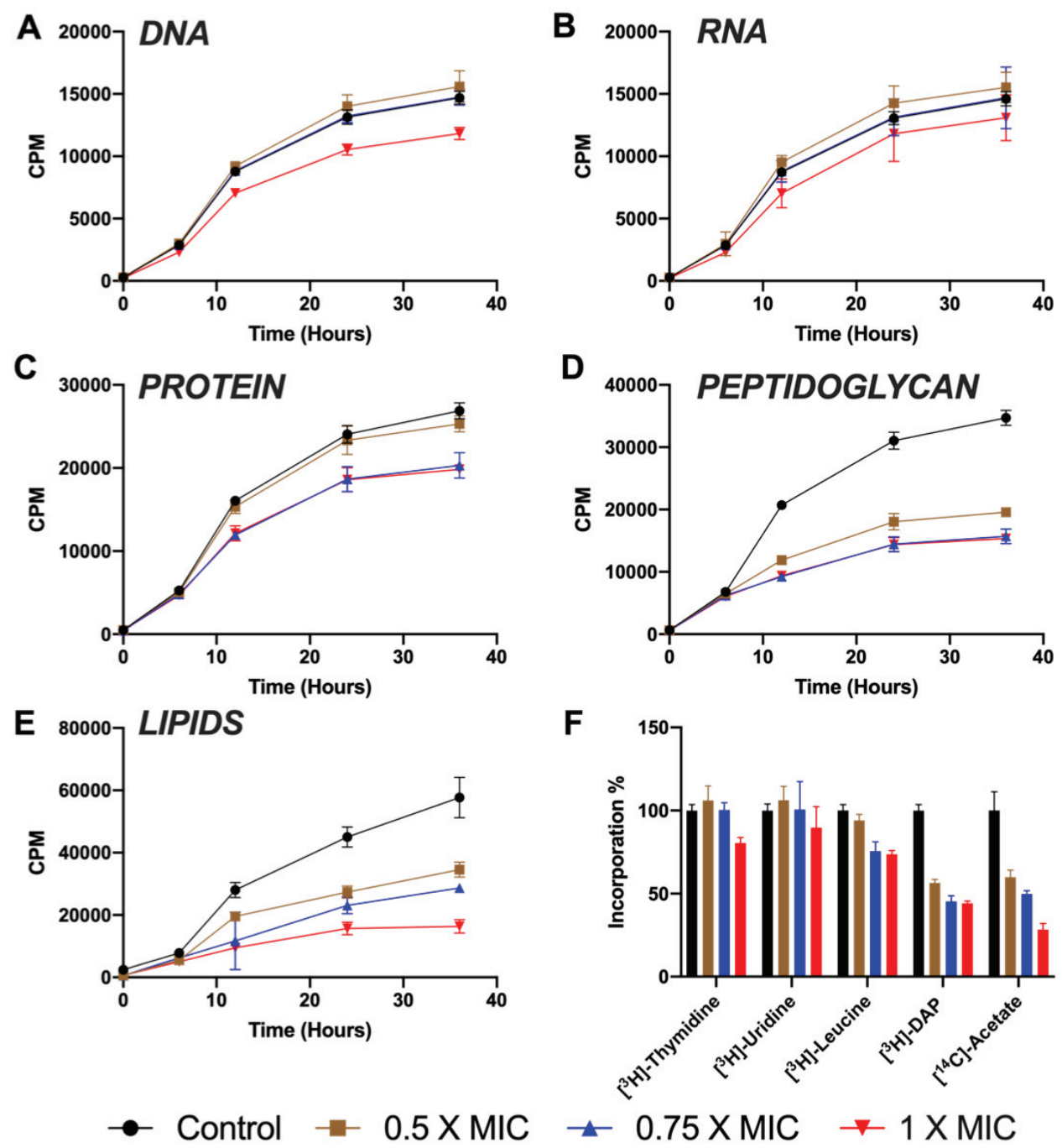

Figure 4. Effect of BGAz-005 on the incorporation of radiolabelled precursors into the major cellular macromolecules of M. smegmatis. The incorporation of (A) $\left[\right.$ methyl- $\left.{ }^{3} \mathrm{H}\right]$ thymidine (for DNA), (B) $\left[5,6-{ }^{3} \mathrm{H}\right]$ uridine (for RNA), (C) L- $\left[4,5-{ }^{3} \mathrm{H}\right]$ leucine (for protein), (D) $\left[{ }^{3} \mathrm{H}\right]$ meso-diaminopimelic acid (for peptidoglycan) and (E) $\left[{ }^{14} \mathrm{C}\right]$ acetic acid (for lipids) were measured over a period of 36 hours. The percentage of incorporation measured at 36 hours is represented in panel F. Each plot and error bars represent the average of three independent experiments.

\section{$B G A q_{-005}$ dysregulates the expression of cell envelope biosynthetic genes}

To explore the mode of action of BGAz-005 using an unsupervised approach, the M. bovis BCG transcriptional response to drug exposure was profiled by RNAseq. A signature consisting of 160 induced and 126 repressed genes was identified after eight hours' exposure to $1 \times$ MIC BGAz-005. The response was comprised of three principal features, namely, inhibition of cell-wall biosynthesis, dysregulation of metal homeostasis and disruption of the respiratory chain (Figure 5). The inhibition of cell-wall synthesis was evidenced by induction of key regulators of cell-wall stress sig $E$ and $m p r A B$, alongside significant upregulation of their regulons (hypergeometric $\mathrm{p}$ value of sigE regulon enrichment $2.47 \times 10^{-17} ;{ }^{32} \mathrm{mpr} A B$ hgp $5.29 \times 10^{-11}$ ). ${ }^{33}$ In contrast to isoniazid and ethambutol where FasII genes are induced by drug exposure, FasII genes (bad $A$, $f a b G 1, \operatorname{inh} A, a c p M)$ alongside mycolic acid synthesis and modification genes (mmaA2, mmaA3, fbpA, fbpB, 
$f b p D$, des $A 2)$ were repressed by BGAz-005 treatment, indicating a different mechanism of BGAz-005 drug action to cell-wall inhibitors currently in use. The functional category (I.H) lipid biosynthesis was significantly repressed by BGAz-005 (hgp $6.52 \times 10^{-5}$ ), and mycolyl-arabinogalactan-peptidoglycan complex biosynthesis was the top pathway dysregulated by BGAz-005 (pathway perturbation score of 3.4). ${ }^{34,35}$ Genes involved in the synthesis of alternative cell-wall factors, sulfolipids (mmpL8, papA1, pks2) and the oleic acid stearoyl-CoA desaturases that produce phospholipids (desA3_1, desA3_2, BCG_3260c/Rv3230c) were induced. ${ }^{36} \mathrm{~A}$ series of metal-responsive regulatory systems were upregulated by BGAz-005 (cmtR, zur, ideR, tcrYX) as well as genes encoding the lipid-bound siderophore mycobactin $(m b t B, m b t C, m b t D)$, representing disruption of metal control systems, likely impacted by loss of cell-wall structure. Induction of redox-inducible $\operatorname{clg} R$ in combination with repression of the dosR regulon (hgp $6.40 \times 10^{-9}$ ) reflected the impact of BGAz-005 on the respiratory chain. ${ }^{37}$ However, unlike many drugs that affect respiration, no differential expression of energy metabolism systems (nuo $A-N$, qcr $A-C$, ctaC-E, yddA-D, narG-J) was observed. ${ }^{38,39}$ Systems implicated in the efflux (mmpL5, mmpS5, BCG_0727/Rv0678, BCG_0728c/Rv0679c) or detoxification (BCG_3184c/Rv3160c, BCG_3185c/Rv3161c, BCG_3186c/Rv3162c) of antimicrobial drugs were also induced by BGAz-005. ${ }^{40}$ Significantly, the efflux pump efp $A$, highly induced by cell-wall targeting drugs isoniazid, ethambutol and benzothiazinone, was not induced by BGAz-005 exposure.

Mapping the drug-responsive gene clusters identified by Boshoff and co-workers revealed significant enrichment of GC-27 and GC-82 representing cell-wall inhibition, ${ }^{41}$ alongside GC-39 (dosR regulon) and GC-108 (iron scavenging). The most similar drug signatures were the phenothiazines, chlorpromazine and thioridazine (hgp $2.83 \times 10^{-14}$ ), disrupting the cell-wall and electron transfer chain, ${ }^{42}$ alongside analogues of ethambutol (hgp $4.98 \times 10^{-13}$ ) 43 and benzothiazinone (hgp $8.00 \times 10^{-8}$ ) targeting arabinose biosynthesis in the mycobacterial cell-wall. ${ }^{44}$ Thus, BGAz-004 and BGAz-005 elicit a transcriptomic response representing major abrogation of normal cell envelope function. 


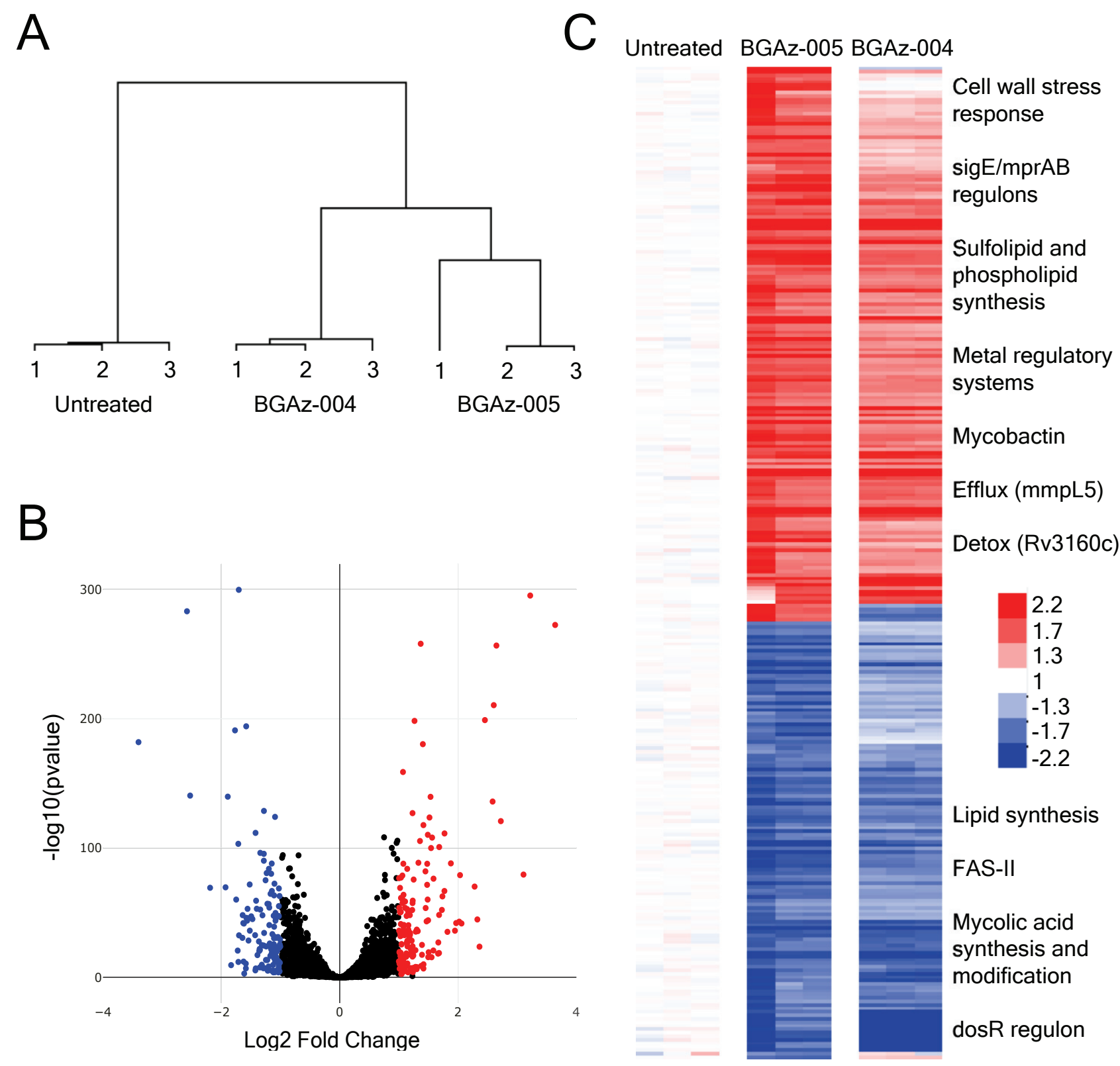

Figure 5. The transcriptional response to BGAz-005 exposure demonstrating inhibition of mycobacterial cell envelope biosynthesis. (A) Cluster diagram of all genes showing similarity of biological replicates and separation of drug-treated from carrier control samples. (B) Volcano plot of $M$. bovis BCG response to BGAz-005, highlighting genes significantly differentially expressed. (C) Heatmap of 286 gene BGAz-005 signature relative to carrier control. Conditions as columns, genes as rows; red colouring highlighting induced genes, blue repressed genes. The BGAz-004 signature is clustered alongside indicating a similar mode of drug action.

\section{$B G A z_{-} 004$ and $B G A z_{-} 005$ significantly alter mycobacterial cell envelope composition}

The results of transcriptomic profiling and whole cell phenotyping support the hypothesis that BGAz-004 and BGAz-005 inhibit aspects of cell envelope biosynthesis. To further investigate the mechanism by which mycobacterial cell envelope lipid composition is affected, actively growing cultures of BCG were exposed to increasing concentrations of BGAz-005 followed by metabolic labelling using $\left[{ }^{14} \mathrm{C}\right]$-acetic acid. 
Autoradiographaphs of cell envelope lipids separated by thin layer chromatography (TLC) revealed that treatment of BCG with BGAz-005 at $0.5 \times$ MIC caused a significant reduction in trehalose monomycolate (TMM) and trehalose dimycolate (TDM) and a complete loss of TMM and TDM at concentrations beyond the MIC (Figure 6A). The formation of cytoplasmic membrane phospholipids (PIMs and CL) remain unaffected (Figure 6A), The analysis of lipids loaded and separated by TLCs that had been normalised for total lipids extracted, revealed an altered lipid profile highlighting the accumulation of an unidentified lipid species that resolves to a relatively high Rf (Lipid species X, Figure 6B). The analysis of mycolic acid methyl esters (MAMES) reveals that both alpha and keto mycolates bound to the cell-wall arabinogalactan (AG) are gradually depleted as BCG is exposed to increasing concentrations of BGAz-005 during active cell culture (Figure 6C). Quantification of the relative abundance of each lipid species highlights the significant depletion of mycolates (either conjugated to trehalose in the form of TMM/TDM or AG) when BGAz-005 is used at a half MIC, whilst other lipids including PI and PIMS remain largely unaffected (Figure 6D). BGAz-004 affects mycobacterial cell envelope lipid biosynthesis in an almost identical manner (Supplementary Figure 3). The immediate and specific arrest in the biosynthesis of TMM and TDM, and as a result, loss of esterified mycolates to AG, strongly supports the hypothesis that BGAz-004 and BGAz-005 inhibit mycobacteria by targeting mycolate biosynthesis. 

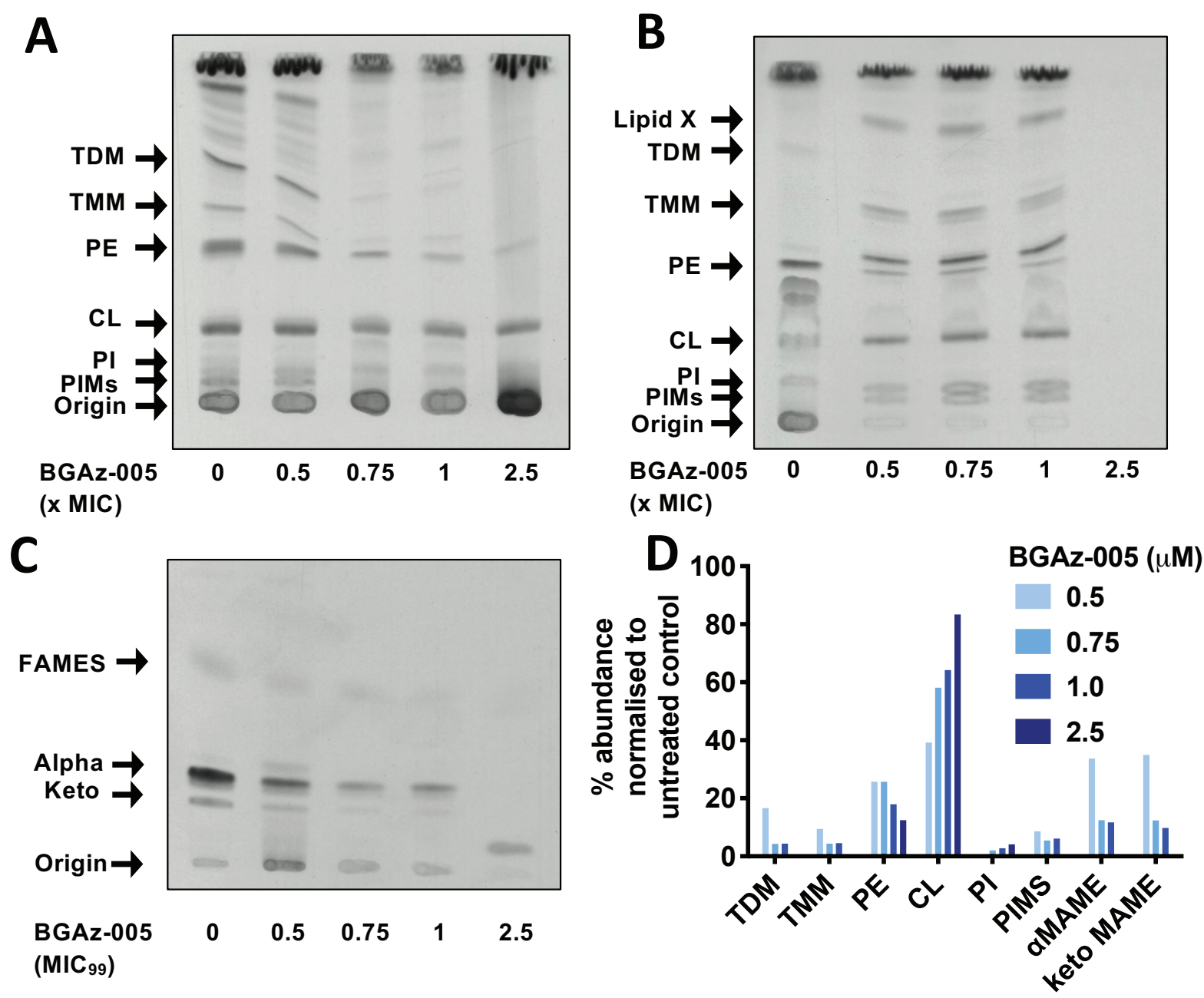

Figure 6. BCG cell envelope lipid analysis upon exposure to BGAz-005. BCG were cultured in 7H9 broth and exposed to increasing concentrations of BGAz005. Lipids were selectively labelled with $\left[{ }^{14} \mathrm{C}\right]$-acetic acid for 12 hours and cell envelope lipids were selectively removed by solvent extraction, separated by TLC (chloroform/methanol/water, 80:20:2, v/v/v), and visualised by autoradiography. A: equal volumes of lipids loaded adjusted for BCG growth; B: equal counts of lipids $(25,000 \mathrm{cpm})$ loaded; C: Mycolic acid methyl ester (MAME) analysis of cell-wall bound mycolates released by $5 \%$ TBAH and separated by TLC (petroleum ether/acetone, 95:5, v/v); D: quantification of BCG lipids from panels A-C by densitometry.

To investigate the effect of BGAz-005 on mycobacterial cell envelope composition and to identify the composition of Lipid-X, actively growing cultures of $M$. smegmatis were exposed to a range of BGAz-005 concentrations, which resulted in a titratable-dependent reduction in the formation of TMM and TDM as observed by staining with MPA and $\alpha$-naphthol (Figure 7A/B), consistent with $\left[{ }^{14} \mathrm{C}\right]$-labelling experiments performed when BGAz-005 was exposed to BCG (Figure 6). M. smegmatis exposed to the highest concentration of BGAz-005, resulted in a significant increase in the relative abundance of free mycolic acid (MA) within the cell envelope (Figure 7C); BGAz004 affects mycobacterial cell envelope lipid biosynthesis in an almost identical manner (Supplementary Figure 4. The gradual reduction of TMM and TDM abundance in $M$. smegmatis and $M$. bovis BCG is a distinct observable phenotype that occurs upon exposure to BGAz005 (Figure 6 and Figure 7; and Supplementary Figures 3 and 4). The separation of solvent extractable lipids by two-dimensional TLC provides further confirmatory evidence that the increasing abundance of Lipid-X 
can be directly attributed to free MA. ${ }^{45}$ This large increase in free mycolic acid, paralleled with the loss of TMM, TDM and arabinogalactan linked mycolates illustrates that BGAz-005 (and BGAz-004) compounds kill mycobacteria by arresting the final stages of mycolate biosynthesis.

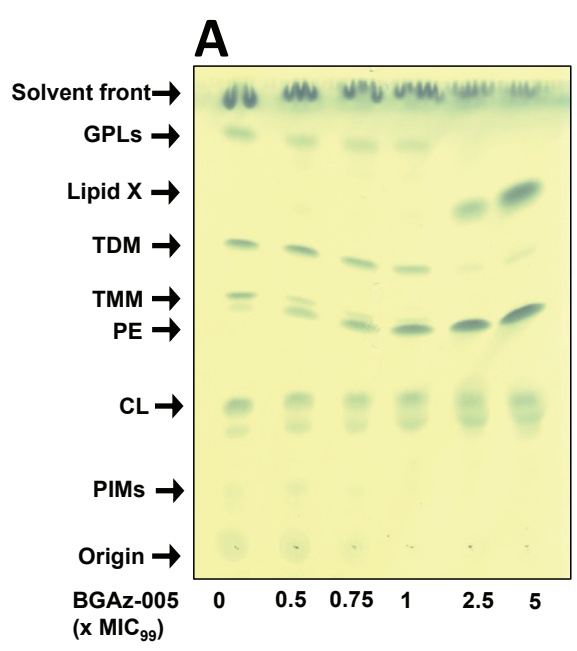

B
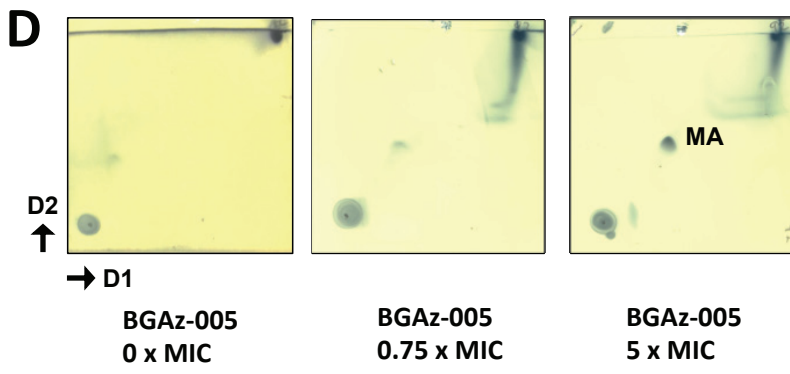

Figure 7. M. smegmatis cell envelope lipid analysis upon exposure to BGAz-005. M. smegmatis were cultured in 7H9 broth, exposed to increasing concentrations of BGAz-005 for six hours and the cell envelope lipids selectively removed by solvent extraction. Equal volumes of lipid adjusted by bacterial growth were separated by TLC (chloroform/methanol/water, 80:20:2, v/v/v), and stained with MPA (A) or alpha-naphthol (B). Equal volumes of lipid adjusted by bacterial growth were separated by TLC (hexane/diethyl ether/acetic acid), 70:30:1, v/v/v and stained with MPA (C). Equal volumes of lipid adjusted by bacterial growth were separated by 2D-TLC (direction 1 chloroform/methanol 96:4, v/v, direction 2 toluene/acetone 80:20, v/v) and stained with MPA (D).

\section{$B G A z_{-0} 04$ and $B G A z_{-005}$ target late-stage mycolic acid biosynthesis enzymes}

The inhibition of mycolate incorporation into the mycobacterial cell-wall, supported by transcriptomic profiling suggests that BGAz-004 and BGAz-005 act by targeting late-stage mycolate biosynthesis (Figure 5, Figure 6 and Figure 7). The accumulation of free mycolic acid upon exposure of BGAz-004 and BGAz-005 at the highest concentrations, suggests that mycolates are being formed but not deposited into the cell-wall (Figure 7 and Supplementary Figure 4). Pks13, MmpL3 and the Ag85 complex (FbpA, FbpB and FbpC) represent a selection of putative enzyme targets of mycolate biosynthesis that could be inhibited by BGAz004 and BGAz-005. Pks13 catalyses the last condensation reaction in mycolate biosynthesis, condensing two fatty acids to form mycolic acids. ${ }^{38}$ It also plays a role in TMM formation through acylation of trehalose. ${ }^{39}$ 
Although essential in mycobacteria, Corynebacterium glutamicum can survive without mycolates, ${ }^{38}$ and so a Pks13 deletion mutant of C. glutamicum was utilised in this study (Supplementary Information). BGAz-005 inhibits corynemycolic acid biosynthesis in C. glutamicum similarly to mycobacteria (Supplementary Figure 6. Both BGAz-002-BGAz-005 retained similar levels of activity in C. glutamicum $\Delta p k s 13$ as in the wild-type strain, implying that Pks13 is not the target (Supplementary Table 2). MmpL3 is the essential membrane transporter responsible for translocating TMM across the cytoplasmic membrane. ${ }^{37,40}$ Treatment of M. bovis BCG harbouring an MmpL3 overexpression vector (pMV261A-mmpL $3^{41}$ ) with BGAz-002-BGAz-005 resulted in no shift in the $\mathrm{MIC}_{99}$ compared to the empty vector (pMV261A) control (Supplementary Table 2). This negates MmpL3 as a potential target of the active BGAz compounds in this study, as overexpression of mmpL3 should result in an increase in MIC as a result of increased copy number and target abundance. The Ag85 complex consists of three essential enzymes with mycolyltransferase activity, responsible for the formation of TMM, TDM, and the covalent attachment of mycolic acids to arabinogalactan. ${ }^{42}$ BCG harbouring the plasmids p'TIC6- $f b p A$, p'TIC6- $f b p B$, and p'TIC6- $f b p C$ overexpressing Ag85A, Ag85B and Ag85C, respectively, were treated with BGAz-004 and BGAz-005. A statistically significantly increase in MIC was seen with FbpB and FbpC, but not FbpA (Figure 8). A two-fold increase in MIC was seen for both BGAz-004 and BGAz-005 upon overexpression of FbpB and FbpC, compared to the three-fold increase seen with known covalent inhibitor Ebselen. ${ }^{46}$ No shift in MIC was seen for FbpA upon Ebselen treatment. Furthermore, M. smegmatis cultured in the presence of Ebselen also resulted in a significant increase of free mycolic acid (MA) within the cell envelope and a concomitant loss of TMM and TDM (Supplementary Figure 5), mirroring the lipid profiles induced by BGAz-004 and BGAz-005 (Figure 7). Collectively, these findings point toward the antigen 85 enzymes as a possible target of the active BGAz compounds of this study. 
A

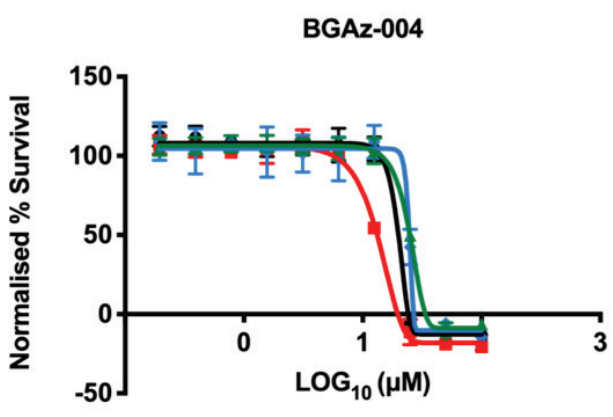

C
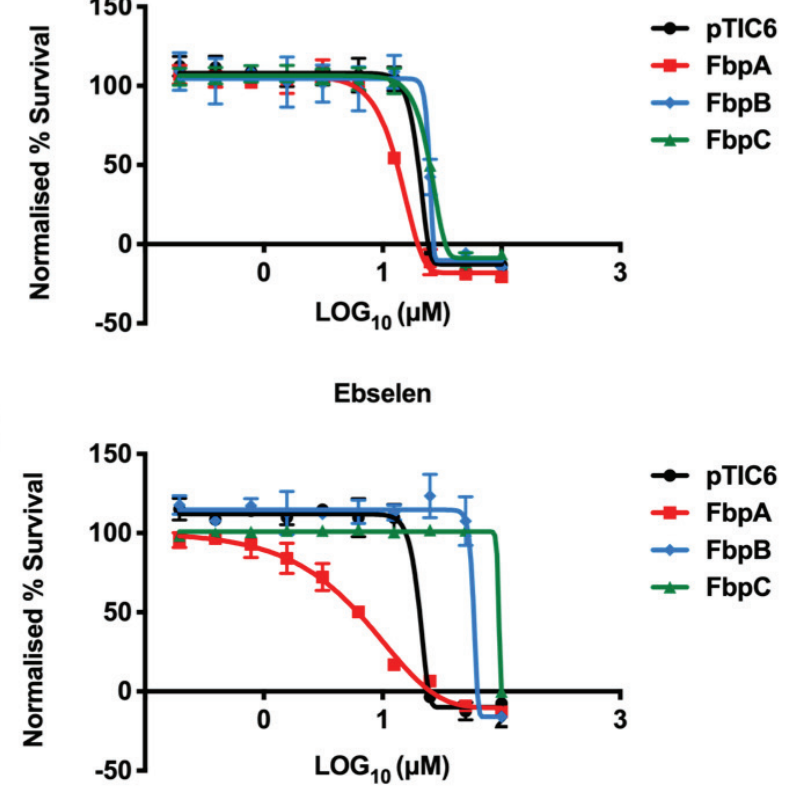
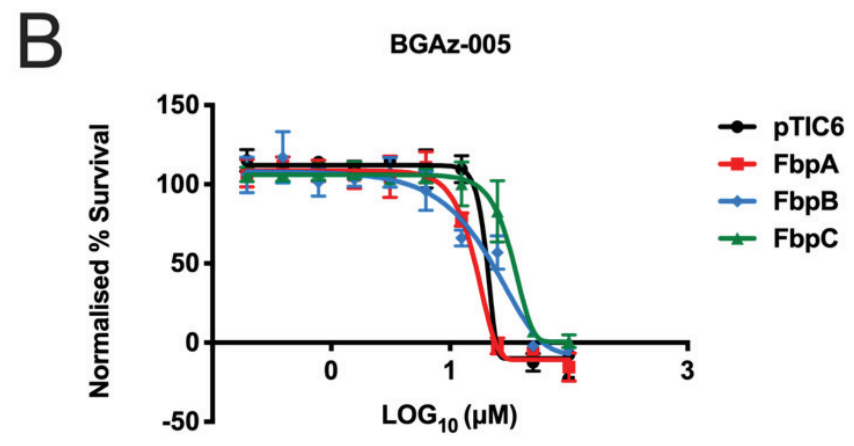

D

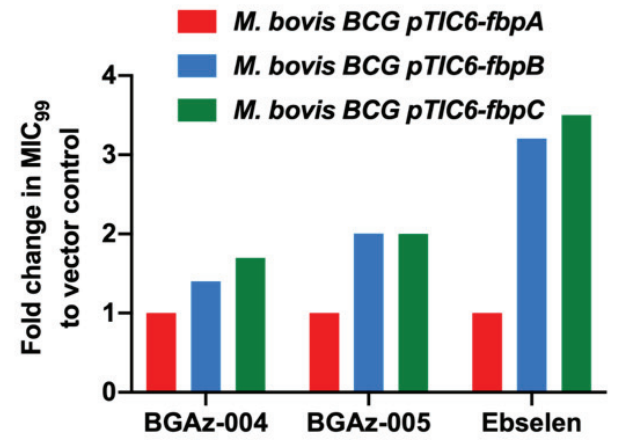

Figure 8. Assessing the MIC shift of BGAz-004 and BGAz-005 against the AG85 complex. MIC values of BGAz-004, BGAz-005 and Ebselen were determined against BCG harbouring overexpression vectors and compared to empty vector controls (pTIC6) in order to identify a shift in MIC against fbpA

(A), fbpB (B), fbpC (C). Fold change in MIC shift (D). The MIC 99 was calculated using an endpoint resazurin assay and the Gomperz equation for MIC determination (GraphPad Prism). Data is of triplicate repeats.

\section{Discussion}

The emergence of multi-drug-resistant TB means that new drugs to treat this disease are desperately required. Any new therapy should meet a number of parameters: it should be effective against MDR-TB; it should be rapidly bactericidal; it should show a novel mechanism of action; and it should possess ADME properties suitable for once a day oral dosing and co-administration with the current TB therapies and anti-HIV agents. ${ }^{1}$ BGAz-002-BGAz-005 display potent inhibitory activity against different mycobacterial species (including virulent and avirulent $M$. tuberculosis reference strains), as well as drug-sensitive and drug-resistant (resistant to isoniazid, rifampicin, pyrazinamide and ethambutol) clinical isolates of $M$. tuberculosis. It is promising that BGAz-002-BGAz-005 retain similar levels of activity between drug-sensitive and drug-resistant clinical isolates of $M t b$; not only do these compounds target MDR-TB, but this also suggests that there is no crossresistance with the current frontline drugs, indicative of a distinct mode of action. In addition to targeting an MDR strain of $M t b$, the active BGAz compounds tested display no detectable resistance against mycobacteria in the laboratory, suggesting that development of clinical resistance to this class of compounds will be slow to occur. Similarly, teixobactin is a cyclic undecapeptide antibiotic that elicits bactericidal activity towards clinically relevant Gram-positive pathogens, also displaying an undetectable frequency of resistance. Teixobactin has a unique mode of action; by binding simultaneously to the cell-wall biosynthetic precursors 
lipid II and Lipid III, this antibiotic inhibits the biosynthesis of peptidoglycan and cell-wall teichoic acids, respectively. ${ }^{47}$

For each of BGAz-002-BGAz-005, the $\mathrm{MBC}$ is within four-fold of the $\mathrm{MIC}_{99}$, demonstrating their bactericidal nature in BCG. Further assessment in $M$. tuberculosis by time-kill viable counts and flow cytometry confirmed the bactericidal activity of BGAz-004 and BGAz-005, with BGAz-005 being significantly bactericidal after six days. The significantly earlier bactericidal activity of BGAz-005 compared to BGAz$\mathbf{0 0 4}$ could be attributed to the increased kinetic solubility of BGAz-005, or it could suggest that BGAz-005 has additional targets. Whilst neither BGAz-004 nor BGAz-005 displayed bactericidal activity as rapidly as the current frontline drug isoniazid, ${ }^{48}$ the slower killing induced by the BGAz compounds tested may prove advantageous in preventing bacterial re-growth. ${ }^{49}$ Previous studies have shown that the rapid, early bactericidal activity of isoniazid results in bacterial regrowth, compared to no regrowth seen with the slower acting bactericidal drugs rifampicin and pyrazinamide. ${ }^{2}$ The longer-acting bactericidal activity of the BGAz compounds tested, combined with the absence of any detectable generation of resistance, suggests that they may be superior to isoniazid, as the potential for tolerance and resistance are very low.

Comprehensive mode of action studies support the hypothesis that the BGAz compounds target mycobacterial cell-wall biosynthesis. Radiolabelled precursor incorporation studies demonstrate that BGAz$\mathbf{0 0 5}$ specifically arrests peptidoglycan and lipid biosynthesis, and transcriptome signatures of BGAz-004- and BGAz-005-treated $M$. bovis BCG reveal significant alterations in cell-wall biosynthetic genes. The mycobacterial cell-wall is a well validated and commonly occurring target amongst anti-tubercular drugs, including frontline drugs isoniazid ${ }^{3}$ and ethambutol, ${ }^{4}$ as well as ethionamide, ${ }^{3}$ SQ109, ${ }^{5}$ and D-cylcoserine. ${ }^{6}$ Whilst the BGAz compounds tested in this study also inhibit cell-wall biosynthesis, they do so without displaying target redundancy against current front line drugs, such as isoniazid. Transcriptomic analysis revealed marked differences between the BGAz-004, BGAz-005, isoniazid and ethambutol, specifically the downregulation of mycolic acid synthesis genes of the FasII system and mycolic acid synthesis and modification genes, and the lack of induction of the efp $A$ efflux pump. Compared to other genome-wide transcriptional studies of anti-tubercular drugs, ${ }^{50}$ the observed differences induced by BGAz compounds suggest that they possess a unique mode of action compared to current chemotherapeutic agents.

To further probe the mechanisms by which the BGAz compounds perturb the mycobacterial cell envelope, the lipid profiles of mycobacteria exposed to increasing concentrations of BGAz-005 were examined. A specific and rapid depletion in TMM and TDM was seen upon BGAz treatment of both M. smegmatis and $M$. bovis BCG, whilst other lipids such as cardiolipin and PIMs remained constant. The effects are more pronounced in BCG due to its increased sensitivity of radiolabelling, but in both instances, there is an almost 
complete arrest in TMM and TDM production by $1 \times$ MIC of BGAz-005. Analysis of the cell-wall bound mycolates revealed a concurrent depletion in MAMEs. The specific loss in mycolates, both non-covalently (TMM and TDM) and covalently (MAMEs) associated, indicates that the BGAz compounds tested target mycolic acid biosynthesis. Mycolates are an essential component of the mycobacterial cell envelope and are targeted by the current drugs isoniazid and ethionamide. ${ }^{51}$ That the BGAz compounds tested target the same biosynthetic pathway as isoniazid corroborates with the flow cytometry analysis, where the staining profiles of BGAz-004 and BGAz-005 were like those of isoniazid, suggesting a similar target. Further cell envelope analysis revealed a pronounced increase in free mycolic acid correlating with increasing concentration of BGAz-005. Typically, the relative abundance of free MA in the cell envelope of planktonically cultured mycobacteria is extremely low. However, previous studies have demonstrated that free MA levels increase significantly when mycobacteria are cultured as a pellicle biofilms ${ }^{52}$ or as non-replicating populations induced by gradual nutrient starvation. ${ }^{53}$ This simultaneous depletion of mycolates conjugated to trehalose and AG, alongside an accumulation of free mycolic acid implies that the mycolates are being synthesised (demonstrated by the accumulation of free mycolate), but are not incorporated into the cell-wall (demonstrated by the loss of TMM, TDM and MAMEs). Thus, unlike isoniazid, the BGAz compounds tested target late-stage mycolic acid biosynthesis and have a mode of action distinct to that of isoniazid and Ethionamide, which target the early stages of mycolate production. ${ }^{3}$ There are several enzyme candidates involved in the latter stages of mycolic acid biosynthesis and incorporation into the cell envelope. Specifically, These encode the polyketide synthase (Pks13) responsible for the last condensation reaction in mycolate biosynthesis, ${ }^{7}$ the essential membrane transporter responsible for translocating TMM across the cytoplasmic membrane (MmpL3), ${ }^{8,9}$ and the mycolyltransferase responsible for the formation of TMM, TDM, and the covalent attachment of mycolic acids to arabinogalactan by the antigen 85 complex enzymes (FbpA, FbpB and $\mathrm{FbpC}),{ }^{10}$ respectively. Target engagement over-expression studies ruled out MmpL3 as a BGAz target, while experiments conducted in C. glutamicum demonstrate that Pks13 is equally not inhibited by these compounds. Guided by evidence obtained from transcriptomic profiling and cell envelope lipid analysis, further target engagement over-expression studies revealed that $\mathrm{FbpB}$ and FpbC afforded moderate protection to BCG exposed to BGAz-004 or BGAz-005. Previous studies have identified FbpA, FbpB and FpbC as druggable targets for the development of new anti-tubercular agents, ${ }^{46,54-56}$ however many of these agents display unfavourable toxicological and PK/PD profiles. In this regard, the BGAz compound series demonstrates an encouraging overall toxicological profile with good absorption, low mitochondrial toxicity and rapid clearance from hepatocytes. In order to reduce the potential of compound-related risk factors a selection of mechanistic screening assays was utilised to identify hazardous and undesirable chemistry in this study. A critical example of such compound liabilities is the blocking of hERG potassium channel. The 
hERG channel is a voltage-gated potassium channel that is expressed in a variety of human tissues such as the brain, thymus, adrenal gland, retina and in cardiac tissue. Any significant blocking of hERG channels by potential drug candidates could have serious off-target effects due to the dysregulation of action potential repolarisation. Furthermore, BGAz-005 displays a remarkable pharmacokinetic profile in mice, with a blood plasma half-life exceeding three days that exceeds the $\mathrm{C}_{\max }$ required to reach a therapeutic dose for MDRTB. Overall, the results presented here demonstrate that the BGAz compound series represents promising novel anti-tubercular agents for further development towards the clinic.

\section{Methods}

\section{Synthesis of BGAz-001-BGAz-005}

Novel racemic 2,4-cis-amino-azetidine derivatives (BGAz-001-BGAz-005) were prepared and purified according to procedures reported for the synthesis of related compounds, for details see supplementary material (Supplementary Scheme 1). ${ }^{16-19}$ Briefly, commercially available aldehydes (S1) and amines (S2) were dissolved in methanol and heated at reflux to afford the corresponding imines (S3). ${ }^{57-59}$ Imines (S3) were isolated and subsequently reacted with in situ prepared allyl zinc reagent to afford homoallyl amine derivatives thereof (S4). The homoallyl amine derivatives (S4) thus obtained were dissolved in acetonitrile and treated with iodine ( 3 equiv.) and sodium bicarbonate (5 equiv.) at temperatures not exceeding $20{ }^{\circ} \mathrm{C}$ resulting in cyclisation to the corresponding 2-iodomethyl azetidine derivatives (S5). Displacement of iodine in derivatives $\mathbf{S} 5$ by the appropriate primary or secondary amines delivered the BGAz series of compounds in four linear steps.

\section{Bacterial strains and growth conditions}

M. smegmatis $\mathrm{MC}^{2} 155$ was cultured at $37^{\circ} \mathrm{C}, 180 \mathrm{rpm}$ in Middlebrook $7 \mathrm{H} 9$ media supplemented with $0.05 \%$ Tween-80, or grown on LB agar. M. bovis BCG (strain Pasteur) was cultured at $37{ }^{\circ} \mathrm{C}$ and $5 \% \mathrm{CO}_{2}$, static, in Middlebrook $7 \mathrm{H} 9$ media supplemented with 0.05\% Tween-80 and 10\% (v/v) BBL Middlebrook OADC enrichment or grown on Middlebrook 7H11 agar supplemented with 10\% (v/v) BBL Middlebrook OADC enrichment.

\section{Determination of MIC and MBC}

The minimum inhibitory concentration $\left(\mathrm{MIC}_{99}\right)$ was determined in 96-well flat bottom, black polystyrene microtiter plates (Greiner) in a final volume of $200 \mu \mathrm{L}$ Compounds were two-fold serially diluted in neat DMSO and added to the microtiter plate at a final concentration of $1 \%$ DMSO. DMSO (1\% in $7 \mathrm{H} 9)$ was used as a positive control and rifampicin as a negative. The inoculum was standardised at $\mathrm{OD}_{600} 0.05$ in 
Middlebrook $7 \mathrm{H} 9$ medium and added to the plate, which was then incubated without shaking at $37{ }^{\circ} \mathrm{C}$ for 24 hours (M. smegmatis) or seven days (M. bovis BCG). Following incubation, $42 \mu \mathrm{L}$ of resazurin $(0.02 \% \mathrm{v} / \mathrm{v}$ in $\mathrm{dH}_{2} \mathrm{O}$ ) was added to each well and incubated for a further two hours (M. smegmatis) or $24 \mathrm{~h}$ (M. bovis $\mathrm{BCG}$ ). Fluorescence was measured (Polar star omega plate reader ex $544 \mathrm{~nm}$, em $590 \mathrm{~nm}$ ) and the data normalised using equation one. The concentration of drug required to inhibit cell growth by $99 \%$ was calculated by nonlinear regression (Gomperz equation for MIC determination, GraphPad Prism).

$$
\left(\frac{x-\bar{x}(\text { negative controls })}{\bar{x}(\text { positive controls })-\bar{x}(\text { negative controls })}\right) \times 100
$$

Equation 1

To determine the minimum bactericidal concentration (MBC), M. smegmatis and M. bovis BCG were grown in the presence of a two-fold serial dilution of compound as above. After a 24-hour (M. smegmatis) or seven-day (M. bovis BCG) incubation, the cells were pelleted and washed with phosphate buffered saline pH 7.2 (PBS). The washed cells were plated onto agar, devoid of compound, and incubated for four days (M. smegmatis) or 21 days $M$. bovis (BCG). The MBC was defined as the lowest concentration of compound for which there was no bacterial growth.

\section{Determination of MIC $C_{\text {lux } 50}$ against autoluminescent M.tb H37Ra}

$\operatorname{AlRa}^{60}$ (Mtb H37Ra::pTYOK) was homogenised with sterile glass beads in a $50 \mathrm{~mL}$ tube containing Middlebook $7 \mathrm{H} 9$ medium (5 mL) plus $0.05 \%$ Tween $80,10 \% \mathrm{v} / \mathrm{v}$ oleic acid albumin dextrose catalase (OADC) supplement (7H9-OADC-Tw). When $\mathrm{OD}_{600}$ reached 0.3-0.5, relative light unit (RLU) count was determined by placing culture $(200 \mu \mathrm{L})$ on the detection hole of the luminometer. When the RLU reached 2 million/mL, the activities of compounds were assessed over a range of three-fold increasing from 0.000001 $\mu \mathrm{g} / \mathrm{mL}$ to $10 \mu \mathrm{g} / \mathrm{mL}$ prepared in $25 \mu \mathrm{L}$ AlRa broth culture (RLU diluted to 2000-4000/25 $\mu \mathrm{L}$ ) grown in $7 \mathrm{H} 9$ broth without Tween 80 . DMSO was used as negative control and isoniazid (INH, $10 \mu \mathrm{g} / \mathrm{mL}, 1 \mu \mathrm{g} / \mathrm{mL}$ and $0.1 \mu \mathrm{g} / \mathrm{mL}$ ) and rifampicin (RIF, $10 \mu \mathrm{g} / \mathrm{mL}, 1 \mu \mathrm{g} / \mathrm{mL}$ and $0.1 \mu \mathrm{g} / \mathrm{mL}$ ) were used as positive controls. RLU counts were determined daily, for four times (daily, i.e. day 0, 1, 2 and 3). The MIClux50 was defined as determined as the lowest concentration that can inhibit $>50 \%$ RLUs compared with that from the untreated controls at day three. ${ }^{61}$

\section{Determination of $M I C_{9 g}$ for clinical M.tb strains}

The previously described resazurin microtiter assay (REMA) plate method was used. ${ }^{62,63}$ Compounds were two-fold serially diluted in CAMR Mycobacterium Medium MOD2 (CMM MOD2). ${ }^{64}$ Individual wells, in a 96-well plate, were inoculated with $1 \times 10^{6} \mathrm{CFUmL}^{-1}$ bacilli and incubated for seven days at $37^{\circ} \mathrm{C}$ with 
agitation $(200 \mathrm{rpm})$. Following this, resazurin solution was added to wells $(0.02 \%$ (w/v) in PBS pH 7.4, supplemented with 5\% Tween 80). ${ }^{65}$ The 96-well plates were incubated at room temperature for six hours. The $\mathrm{OD}_{570 \mathrm{~nm}}$ of each well was recorded using a Tecan Sunrise plate reader. The minimum inhibitory concentration $\left(\mathrm{MIC}_{99}\right)$ was calculated using a modified Gompertz function. ${ }^{66}$ The optical density measurements for each drug concentration was compared to vehicle control to determine the percentage reduction in bacterial optical density.

\section{Physiochemical and toxicological analysis}

\section{Kinetic Solubility}

Compounds were solubilised (10 $\mathrm{mM}$ in DMSO) and diluted in phosphate buffered saline (pH 7.4) into a seven-point curve $(0.2-100 \mu \mathrm{M})$ and incubated for five minutes at $25{ }^{\circ} \mathrm{C}$ with shaking (final DMSO concentration 1\%). The turbidimetry was assessed at each of the seven concentrations using UV spectrophotometry at $620 \mathrm{~nm}$, and the LogS was converted into the estimated solubility (S) using the equation $S=10^{\log S}$. Nicardipine hydrochloride was used as a control compound. All experiments were performed in triplicate.

\section{Mouse PPB}

Compounds were solubilised (10 mM in DMSO) and Rapid Equilibrium Dialysis (RED) was used to measure the percentage binding to mouse plasma protein of the BGAz compounds at a final concentration of $5 \mu \mathrm{M}$. The BGAz compound was incubated in 100\% mouse plasma and dialysed against buffer in a RED device for four hours at $37{ }^{\circ} \mathrm{C}$ in a $5 \% \mathrm{CO}_{2}$ incubator, with continuous shaking at $200 \mathrm{rpm}$. Samples were matrix matched and analysed by LC-MS/MS against a six-point standard curve prepared with 100\% plasma. All experiments were performed in triplicate.

\section{Mouse Microsomal Clearance}

Compounds were solubilised (10 mM in DMSO). $1 \mu \mathrm{M}$ of BGAz compound was incubated with $0.5 \mathrm{mg} / \mathrm{mL}$ mouse microsomes in the presence or absence of the Phase 1 cofactor NADPH $(1 \mathrm{mM})$ at $37^{\circ} \mathrm{C}$ for 0,5 , 10, 15, and 30 minutes. The disappearance of the BGAz compound was assessed by LC-MS/MS. All experiments were performed with two replicates per compound and were validated by the inclusion of up to three species-specific control compounds. Data output consists of mean intrinsic clearance (CLint) and halflife $\left(t_{1 / 2}\right)$ measurements.

\section{Mouse Hepatocyte Clearance}

Compounds were solubilised (10 mM in DMSO). $1 \mu \mathrm{M}$ of BGAz compound was incubated with $0.5 \times 10^{6}$ cells/mL mouse hepatocytes at $37^{\circ} \mathrm{C}$ for $0,10,20,30,45$ and 60 minutes. The disappearance of the BGAz compound in the presence and absence of hepatocytes was assessed using LC-MS/MS. All experiments were 
performed with two replicates per compound and were validated by the inclusion of up to three speciesspecific control compounds. Data output consists of mean intrinsic clearance ( $\left.\mathrm{CL}_{\mathrm{int}}\right)$ and half-life $\left(\mathrm{t}_{1 / 2}\right)$ measurements.

Caco-2 and efflux

Compounds were solubilised (10 mM in DMSO) and the CacoReady ${ }^{\mathrm{TM}}$ Kit from ReadyCell S.L. (Barcelona, Spain) was used to determine compound permeability. Differentiated and polarized Caco-2 cells (21-day system) were plated on a 96-transwell permeable system as a single monolayer to allow for automated high throughput screening of compounds, and $10 \mu \mathrm{M}$ BGAz compound was added to the system in HBSS buffer $(\mathrm{pH} 7.4)$ and incubated for two hours at $37^{\circ} \mathrm{C}$ in a $\mathrm{CO}_{2}$ incubator. Lucifer yellow was used as a cell monolayer integrity marker. Drug transport was assessed in both directions (apical to basolateral (A-B) and basolateral to apical (B-A)) across the cell monolayer. The buffer used for the assay does not include HEPES, so as to minimise the inhibitory effect on uptake transporters. ${ }^{67}$ The BGAz compound concentrations were quantified using a calibration curve following analysis by LC-MS/MS, and the apparent permeability coefficient (Papp) and efflux ratio of the compound across the monolayer were calculated. The efflux ratio is used as an indicator of active efflux. The permeability coefficient $\left(P_{a p p}\right)$ was calculated from the following equation:

$$
P_{a p p}=\left(\frac{d Q / d t}{C_{0} \times A}\right)
$$

Equation 2

Where $\mathrm{dQ} / \mathrm{dt}$ is the amount of compound in basal (A-B) or apical (B-A) compartment as a function of time $(\mathrm{nmol} / \mathrm{s}) \cdot \mathrm{C}_{0}$ is the initial concentration in the donor (apical or basal) compartment (Mean of $\mathrm{T}=0$ ) $(\mathrm{nmol} / \mathrm{mL})$ and $\mathrm{A}$ is the area of the transwell $\left(\mathrm{cm}^{2}\right)$.

The efflux ratio was then calculated as:

$$
\frac{P_{a p p(B t o A)}}{P_{a p p}(A t o B)}
$$

All experiments were performed in triplicate and the MDR1 efflux markers Digoxin, quinidine and propranolol were used as positive controls.

\section{Pharmacokinetic studies}

Methods for combined single dosing (BGAz001-005) and dosing of single compounds multiple times (BGAz004 and BGAz005) are described in detail in a supplementary file (Supplementary PK Data). 


\section{Cytochrome P450 activities}

Compounds were solubilised to $10 \mathrm{mM}$ in DMSO. The BGAz compounds were incubated at concentrations of 0.003, 0.009, 0.03, 0.08, 0.25, 0.74, 2.2, 6.7 and $20 \mu \mathrm{M}$ with CYP1A2, CYP2C9, CYP2C19 and CYP2D6 at $37^{\circ} \mathrm{C}$ in the presence of the drug-like probe substrate HLM the Phase 1 cofactor NADPH $(1 \mathrm{mM})$. The formation of metabolites of the drug-like probe substrates in the absence and presence of BGAz compound was monitored by LC-MS/MS, and the $\mathrm{IC}_{50}$ value determined. All assays had two replicates per compound and included a positive control inhibitor.

\section{HepG2 mitochondrial dysfunction}

Compounds were solubilised to $30 \mathrm{mM}$ in DMSO. The BGAz compounds were added to the HepG2 cell model in a 96-well microplate in half log dilutions from $100 \mu \mathrm{M}-0.0003 \mu \mathrm{M}$ (final DMSO concentration $0.3 \%$ ) using both glucose (DMEM consisting of $25 \mathrm{mM}$ glucose) and galactose (DMEM consisting of 10 $\mathrm{mM}$ galactose) media. The compounds were incubated with the cell line or 24 hours at $37^{\circ} \mathrm{C}$ in a humidified $\mathrm{CO}_{2}$ tissue culture incubator, followed by cell viability staining with MTT (3-(4,5-dimethylthiazol-2-yl)-2.5diphenyltetrazolium bromide) conversion to Formazan product, determined by absorbance measurement. The cell viability $\mathrm{IC}_{50}$ was determined in HepG2 glucose and metabolism modified HepG2 galactose, and the fold change difference between the Glu/Gal IC $\mathrm{IC}_{50}$ was determined. All experiments were performed in duplicate with the mitochondrial toxicity controls rotenone and Antimycin A, and the cytotoxin control tamoxifen.

\section{bERG cardiotoxicity function}

Compounds were solubilised to $30 \mathrm{mM}$ in DMSO before dilution in PBS to $300 \mathrm{mM}$. A further three-fold on-board dilution resulted in a final top BGAz compound concentration of $100 \mathrm{mM}$. Eight-point concentration-response curves were generated using 3.16-fold serial dilutions from the top test concentration. Electrophysiological recordings were made from a Chinese Hamster Ovary cell line stably expressing the full length hERG channel. Single cell ionic currents were measured in the perforated patch clamp configuration $\left(100 \mu \mathrm{gmL} \mathrm{m}^{-1}\right.$ amphotericin) at room temperature $\left(21-23^{\circ} \mathrm{C}\right) \mathrm{using}$ an IonWorks Quattro instrument (Molecular Devices). The internal solution contained (mM): $140 \mathrm{KCl}, 1 \mathrm{MgCl}, 1 \mathrm{EGTA}, 20$ HEPES and was buffered to $\mathrm{pH}$ 7.3. The external solution (phosphate-buffered saline, PBS) contained (mM): $138 \mathrm{NaCl}, 2.7 \mathrm{KCl}, 0.9 \mathrm{CaCl}_{2}, 0.5 \mathrm{MgCl}_{2}, 8 \mathrm{Na}_{2} \mathrm{HPO}_{4}, 1.5 \mathrm{KH}_{2} \mathrm{PO}_{4}$ buffered to pH 7.4. Cells were clamped at a holding potential of $-70 \mathrm{mV}$ for $30 \mathrm{~s}$ and then stepped to $+40 \mathrm{mV}$ for one second. This was followed by a hyperpolarising step of one second to $-30 \mathrm{mV}$ to evoke the hERG tail current. Currents were measured from the tail step and referenced to the holding current. Compounds were then incubated for 34 minutes prior to a second measurement of the hERG signal using an identical pulsetrain. 


\section{Mycobacterial time kill experiments}

BGAz-004, BGAz-005 and isoniazid were two-fold serially diluted in $100 \mu \mathrm{L}$ CMM MOD2 medium from 96-3 $\mu \mathrm{M}, 96-3 \mu \mathrm{M}$ and 29.2-0.9 $\mu \mathrm{M}$ respectively, vehicle control (0.1\% DMSO) was included in all experimentation. Individual wells of a 96-well micro-titre plate were inoculated to a starting bacterial titre of $1 \times 10^{6} \mathrm{CFUmL}^{-1}$. Micro-titre plates were incubated for $0,2,6,10$ and 14 days at $37^{\circ} \mathrm{C}$ with agitation $(200$ $\mathrm{rpm})$. The bacterial titre at each time point was enumerated via outgrowth of bacilli on solid media for three weeks at $37{ }^{\circ} \mathrm{C}$ via a method adapted from Miles and Misra ${ }^{26}$ where triplicate $20 \mu \mathrm{L}$ spots of bacterial culture are spotted onto Middlebrook 7H10 agar for each ten-fold serial dilution. Statistical analyses of data were performed using a factorial ANOVA and post-hoc Tukey's honestly significant difference test.

\section{Bacterial staining and flow cytometry analyses}

The method reported by Hendon-Dunn et al. $2016^{27}$ was used to analyse stained cell populations by flow cytometry $100 \mu \mathrm{L}$ of $M$. tuberculosis $\mathrm{H} 37 \mathrm{Rv}$ from each antibiotic-incubation, at each time-point, was transferred to a micro-titre plate in quadruplicate and incubated in the dark for one hour at $37^{\circ} \mathrm{C}$ with either no dye added, $20 \mu \mathrm{M}$ calcein violet (CV-AM), $20 \mu \mathrm{M}$ sytox green (SG), or both $20 \mu \mathrm{M}$ CV-AM and $20 \mu \mathrm{M}$ SG. These incubations were then spun by centrifugation and the supernatant was removed. The cells were fixed with 4\% formaldehyde (v/v in water) for one hour. The stained bacteria were examined using a Cytoflex $\mathrm{S}$ (Beckman Coulter) flow cytometer. Lasers with excitatory wavelengths of $488 \mathrm{~nm}$ and $405 \mathrm{~nm}$ were used. SG fluorescence emission (excitation and emission, $488 \mathrm{~nm}$ and $523 \mathrm{~nm}$, respectively) was detected in the FIT-C channel (530/40 BP), and CV-AM fluorescence (excitation and emission, $400 \mathrm{~nm}$ and $452 \mathrm{~nm}$, respectively) was detected in the PB-450 channel (450/50 BP). A quadrant gating strategy was used, ${ }^{27}$ briefly, 10,000 single-cells events were gated upon using a two-parameter dot plot of forward scatter height versus forward scatter area. From gated single-cells, the percentages of the total cell population residing in each polygonal population gate (P1: CV-AM-/SG-, P2: CV-AM+/SG', P3: CV-AM+/SG+, P4: CV-AM-/SG ${ }^{+}$) were obtained. Statistical analysis of data was performed using a Student's $t$ test.

\section{Inhibition of Macromolecular Synthesis.}

Inhibition of macromolecular biosynthesis was assayed by measuring the incorporation of radiolabelled precursors into DNA, RNA, protein, peptidoglycan and fatty acids in the 10\% trichloroacetic acid (TCA) extracts of cells exposed to azetidines. M. smegmatis were cultured in $7 \mathrm{H} 9$ media supplemented with $0.05 \%$ Tween-80 and grown to an $\mathrm{OD}_{600}$ of 0.4 . Cultures $(5 \mathrm{~mL})$ were then transferred into sterile glass tubes and preincubated with $0 \times, 0.5 \times, 0.75 \times$ and $1 \times \mathrm{MIC}_{99}$ azetidines for one hour at $37^{\circ} \mathrm{C}$ with shaking. After preincubation, $10 \mu \mathrm{L}$ of $500 \mathrm{nCi} / \mu \mathrm{L}\left[\right.$ methyl- $\left.{ }^{3} \mathrm{H}\right]$ thymidine, $500 \mathrm{nCi} / \mu \mathrm{L}\left[5,6{ }^{3} \mathrm{H}\right]$ uridine, $500 \mathrm{nCi} / \mu \mathrm{L}$ L- $[4,5-$ $\left.{ }^{3} \mathrm{H}\right]$ leucine, $500 \mathrm{nCi} / \mu \mathrm{L}\left[{ }^{3} \mathrm{H}\right]$ meso-diaminopimelic acid and $500 \mathrm{nCi} / \mu \mathrm{L}\left[{ }^{14} \mathrm{C}\right]$ acetic acid were added to cultures 
to measure synthesis of DNA, RNA, protein, peptidoglycan and lipids, respectively. All cultures were incubated for 36 hours and $100 \mu \mathrm{L}$ samples were sacrificed at $6 \mathrm{~h}, 12 \mathrm{~h}, 24 \mathrm{~h}$ and $36 \mathrm{~h}$ time points by the addition of $50 \mu \mathrm{L} \mathrm{30 \%}$ TCA $/ 70 \%$ ethanol in Eppendorf tubes. Tubes were incubated at room temperature for $60 \mathrm{~min}$ to allow for precipitation of macromolecular material. Samples were individually vacuum filtered using $0.025 \mu \mathrm{m}$ membrane filters (VSWP01300, MF-Millipore) that were prewashed with $500 \mu \mathrm{L} 70 \%$ ethanol. Samples were washed with $3 \times 500 \mu \mathrm{L} 5 \%$ TCA followed by $2 \times 95 \%$ ethanol. Filter papers were dried and combined with $5 \mathrm{~mL}$ scintillation fluid before measuring radioactive counts.

\section{Transcriptomic profiling by $R \mathbf{N A}$-seq analysis}

M. bovis $\mathrm{BCG}$ was cultured to an $\mathrm{OD}_{600}$ of 0.4 before exposure to $1 \times \mathrm{MIC}_{99}$ concentrations of BGAz-004 or BGAz-005 for eight hours in three biological replicates, then compared to carrier control-treated bacilli. Cells were pelleted, flash frozen in liquid nitrogen and stored at $-80{ }^{\circ} \mathrm{C}$. Pellets were resuspended in lysozyme $(600$ $\mu \mathrm{L}, 5 \mathrm{mg} / \mathrm{mL})$ and $\beta$-mercaptoethanol $(7 \mu \mathrm{L} / \mathrm{mL})$ in TE buffer and lysed by bead beating at $6 \mathrm{~m} /$ minute $(1$ $\times 45$ seconds). Samples were subjected to further bead beating $(3 \times 45$ seconds $)$ following the addition of $(60 \mu \mathrm{L}, 10 \%$ SDS). Sodium acetate pH $5.2(3 \mathrm{M}, 60 \mu \mathrm{L})$ and acidified phenol pH $4.2(726 \mu \mathrm{L})$ were added and the tubes mixed well by inversion. Samples were incubated at $65{ }^{\circ} \mathrm{C}$ for five minutes and centrifuged for five minutes at $18,000 \times g$. The upper aqueous phase was transferred to a fresh tube and an equal volume of acid phenol pH 4.2 added and mixed well by inversion. Following heating $\left(65^{\circ} \mathrm{C}\right.$ for two minutes) and centrifugation (five minutes at 18,000 $\times g$ ), the upper aqueous phase was once more transferred to a fresh tube and an equal volume of chloroform:isoamyl alcohol $(24: 1 \mathrm{v} / \mathrm{v})$ added. The sample was mixed well by inversion and centrifuged at 18,000 $\times g$ for five minutes. The upper aqueous phase was transferred to a fresh tube and a $1 / 10$ volume of sodium acetate ( $3 \mathrm{M}, \mathrm{pH}$ 5.2) and three volumes of $100 \%$ ethanol were added. Samples were incubated at $-20{ }^{\circ} \mathrm{C}$ overnight, centrifuged for ten minutes $\left(4{ }^{\circ} \mathrm{C}, 14,000 \times g\right)$ and the supernatant removed. Ethanol $(70 \%$ in water, $500 \mu \mathrm{L})$ was added to the pellet and centrifuged for ten minutes $\left(4{ }^{\circ} \mathrm{C}, 14,000 \times g\right)$. The supernatant was removed, the pellet air-dried, and the extracted RNA resuspended in of RNase-free $\mathrm{dH}_{2} \mathrm{O}(40 \mu \mathrm{L})$. DNase treatment was performed using the TURBO DNA-free kit (Invitrogen). Briefly, a $0.1 \times$ volume of $10 \times$ TURBO DNase buffer was added to the RNA, along with TURBO DNase enzyme ( $1 \mu \mathrm{L}$ of enzyme stock). The sample was incubated for 30 minutes at $37^{\circ} \mathrm{C}$, an additional TURBO DNase enzyme ( $1 \mu \mathrm{L}$ of enzyme) was added, and the sample incubated for another 30 minutes. DNase inactivation reagent ( 0.2 volumes) were added, and the sample incubated at room temperature for five minutes. Following centrifugation at $10,000 \times g$ for 1.5 minutes, the supernatant containing the RNA was transferred to a fresh tube and stored at $-80{ }^{\circ} \mathrm{C}$. The purified RNA was quantified, depleted of rRNA and library-prepped before sequencing by Illumina HiSeq $(150 \times 2$ paired end $)$ by Genewiz Ltd. Adapter 
sequences and poor-quality reads were removed using Trimmomatic v.0.36, before mapping to the Mycobacterium bovis BCG Pasteur 1173P2 genome using Bowtie2 aligner v.2.2.6. Gene expression was quantified using FeatureCounts from the Subread package v.1.5.2. Differentially expressed genes were identified with the DESeq2 R package, normalised by RLE method, and using the Wald test with Benjamini and Hochberg multiple testing correction. Genes with an adjusted p-value $<0.05$ and $\log 2$ fold change $>1$ were considered to be differentially expressed. Significantly enriched signatures, with updated genome annotation, ${ }^{68,69}$ were identified using the hypergeometric function comparing to published drug responses ${ }^{40,41}$ or mapped to metabolic pathways. ${ }^{34,35}$ Genes significantly differentially expressed in response to BGAz-004 and BGAz-005 are detailed in Supplementary Material. Fully annotated RNA-seq data will be deposited in ArrayExpress; accession number to be provided.

\section{Radioisotope labelling of lipids and analysis}

Cells were grown to an $\mathrm{OD}_{600} 0.5$, treated with compound and grown for six hours (M. smegmatis) or overnight (M. bovis BCG). For radio labelling experiments, $1 \mu \mathrm{Ci} \mathrm{mL}^{-1}{ }^{14} \mathrm{C}$ acetic acid was then added followed by a 16hour incubation. Cells were harvested and extracted using chloroform:methanol:water (10:10:3, v/v/v, 2 $\mathrm{mL}$ ) for two hours at $50^{\circ} \mathrm{C}$. Following centrifugation, the organic extracts were combined with chloroform and water $(1.75 \mathrm{~mL}$ and $0.75 \mathrm{~mL}$ respectively). The lower organic phase containing associated lipids was recovered, washed twice with chloroform:methanol:water $(3: 47: 48, \mathrm{v} / \mathrm{v} / \mathrm{v}, 2 \mathrm{~mL})$ and dried with nitrogen gas. Samples were resuspended in chloroform:methanol $(2: 1, \mathrm{v} / \mathrm{v}, 200 \mu \mathrm{L})$ and OD adjusted volumes were subjected to thin-layer chromatography (TLC) analysis. Cell-wall associated lipids were visualised by either heating TLC plates after treatment with molybdophosphoric acid (MPA) in ethanol (5\% w/v) or alphanaphthol in ethanol $(5 \% \mathrm{w} / \mathrm{v})$, or by autoradiography by exposure of Kodak BioMax MR film.

Cell-wall bound lipids from the de-lipidated extracts from the above extraction were released by the addition of a solution of tetra-butyl ammonium hydroxide (TBAH) (5\% m/v, $2 \mathrm{~mL})$ followed by a 16-hour incubation at $100{ }^{\circ} \mathrm{C}$. Water $(2 \mathrm{~mL})$, dichloromethane $(4 \mathrm{~mL})$ and iodomethane $(200 \mu \mathrm{L})$ were then added and mixed thoroughly for $30 \mathrm{~min}$. The organic phase was recovered following centrifugation and washed with water (3 $\times 4 \mathrm{~mL}$ ), dried and resuspended in diethyl ether $(4 \mathrm{~mL})$. After sonication and centrifugation, the supernatant was dried and resuspended in dichloromethane. Equivalent aliquots of the samples were subjected to TLC in petroleum ether:acetone (95:5, v/v) and visualised by MPA and heat or autoradiography.

\section{Target gene overexpression studies}

The constructs pMV261_mmpL3 and pVV16_trp $A B,{ }^{70,71}$ including the empty pMV261 and pVV16 vectors, were electroporated into $M$. bovis BCG as previously described, and the $\mathrm{MIC}_{99}$ determined as described above. 
The constructs p'TIC6_fbp $A$, p'TIC6_fbpB and p'TIC6_fbpC were synthesised by GenScript Ltd by inserting the coding regions of the $M$. tuberculosis $\mathrm{H} 37 \mathrm{Rv}$ genes into the vector pTIC6, which encodes kanamycin selection. The constructs and empty pTIC6 vector were electroporated into M. bovis BCG. Following induction of gene expression with $50 \mathrm{ng} / \mathrm{mL}$ of anhydrotetracycline for 24 hours, the $\mathrm{MIC}_{99}$ was determined as described above.

\section{Supplementary material}

A supplementary file containing a detailed description of author contributions, general methods, chemical and biological general procedures, synthetic chemistry protocols for the synthesis of BGAz001-BGAz005, NMR spectrums, details of mass spectrometry analysis and additional corresponding references. ${ }^{72,73}$

\section{Acknowledgements}

LJA and JSF are grateful for the support underpinning much of this study from MRC Confidence in Concepts and EPSRC follow-on fund schemes. The University of Birmingham are thanked for support, including travel funds permitting AY, XL and YC to undertake training placements at GIBH. JSF is grateful to the Royal Society for training provided as a result of a previous Industrial Fellowship and the EPSRC for previous funding (EP/J003220/1). Funding for part of this study was received from Department of Health Grant in Aid. This work was supported by the National Mega-project of China for Innovative Drugs (2019ZX09721001-003-003) and by the Chinese Academy of Sciences Grant (154144KYSB20190005). TZ received a Science and Technology Innovation Leader of Guangdong Province (2016TX03R095) award. SJW and AG thank the National Centre for the Replacement, Refinement and Reduction of Animals in Research (NC3Rs) for grant support (NC/R001669/1). Qiong Pan (GIBH), Jingfang Xiong (GIBH) and Miaoqin She $(\mathrm{GIBH})$ are thanked for conducting aspects of the PK/PD studies of this report. Dr Chi Tsang (UoB), Dr Peter Ashton (UoB) and Jiajia Wei $(\mathrm{GIBH})$ are thanked for helpful discussions and practical support with aspects of mass spectrometry. Dr Cécile S. Le Duff (UoB) and Dr Neil Spencer (UoB) gave advice on aspects of NMR spectroscopy underpinning the preliminary or previously reported, findings. Yingxue Liu $(\mathrm{GIBH})$ is thanked for help with purification of final products by HPLC where required.

\section{Competing interests}

A patent application disclosing aspects of this study has been filed by the University of Birmingham. The views expressed in this publication are those of the authors and not necessarily those of Public Health England, or the Department of Health. The authors declare no other competing interests. 


\section{Author contributions}

Corresponding authors: LJA Biological aspects including mode of action; JSF Synthetic chemistry aspects including azetidine design and synthesis; CN Medicinal chemistry aspects; JB assessments of compound activity against $M$. tuberculosis; SW analysis of RNA seq data and transcriptomic profiling. Joint first authors: AL examined biological activity, determined MIC values, probed target/mechanism and elucidated aspects of the mode of action; YC contributed to development of methodology for the synthesis of azetidine derivatives and transferred knowledge between teams. All authors contributed critically to devising and executing aspects of this research. A detailed and comprehensive description of author contributions is defined in the associated supplementary material.

\section{References}

World Health Organisation: Global Health Observatory (GHO) data <www.who.int/gho/tb/en/> (Date accessed, 30/06/2020). Global tuberculosis report 2019. World Health Organization: Geneva, Licence: CC BY-NC-SA 3.0 IGO (2019).

Combs, D. L., O'Brien, R. J. \& Geiter, L. J. USPHS Tuberculosis Short-Course Chemotherapy Trial 21: Effectiveness, Toxicity, and Acceptability: The Report of Final Results. Ann. Intern. Med. 112, 397-406, doi:10.7326/0003-4819-76-3-112-6-397 (1990).

Shah, N. S. et al. Worldwide emergence of extensively drug-resistant tuberculosis. Emerg. Infect. Dis. 13, 380-387, doi:10.3201/eid1303.061400 (2007).

Sharma, S. K. \& Mohan, A. Multidrug-Resistant Tuberculosis: A Menace That Threatens To Destabilize Tuberculosis Control. Chest 130, 261-272, doi:10.1016/S0012-3692(15)50981-1 (2006).

Zumla, A., Nahid, P. \& Cole, S. T. Advances in the development of new tuberculosis drugs and treatment regimens. Nat. Rev. Drug Discov. 12, 388-404, doi:10.1038/nrd4001 (2013).

Ma, Z., Lienhardt, C., McIlleron, H., Nunn, A. J. \& Wang, X. Global tuberculosis drug development pipeline: the need and the reality. The Lancet 375, 2100-2109, doi:10.1016/S0140-6736(10)60359-9 (2010).

Raymer, B. \& Bhattacharya, S. K. Lead-like Drugs: A Perspective. J. Med. Chem. 61, 10375-10384, doi:10.1021/acs.jmedchem.8b00407 (2018).

Lovering, F. Escape from Flatland 2: complexity and promiscuity. MedChem Comm 4, 515-519, doi:10.1039/C2MD20347B (2013).

Lovering, F., Bikker, J. \& Humblet, C. Escape from Flatland: Increasing Saturation as an Approach to Improving Clinical Success. J. Med. Chem. 52, 6752-6756, doi:10.1021/jm901241e (2009).

Yang, Y., Chen, H., Nilsson, I., Muresan, S. \& Engkvist, O. Investigation of the Relationship between Topology and Selectivity for Druglike Molecules. J. Med. Chem. 53, 7709-7714, doi:10.1021/jm1008456 (2010).

for Their Exclusion in Bioassays. J. Med. Chem. 53, 2719-2740, doi:10.1021/jm901137j (2010).

Baell, J. B. \& Nissink, J. W. M. Seven Year Itch: Pan-Assay Interference Compounds (PAINS) in 2017_Utility and Limitations. ACS Chem. Biol. 13, 36-44, doi:10.1021/acschembio.7b00903 (2018).

Colomer, I. et al. A divergent synthetic approach to diverse molecular scaffolds: assessment of lead-likeness using LLAMA, an open-access computational tool. Chem. Commun. 52, 7209-7212, doi:10.1039/C6CC03244C (2016).

Synthetic chemistry methodology projects within the School of Chemistry at the University of Birmingham initially provided $\sim 200$ compounds suitable for biological screening.

Feula, A. et al. Synthesis of azetidines and pyrrolidines via iodocyclisation of homoallyl amines and exploration of activity in a zebrafish embryo assay. Org. Biomol. Chem. 11, 5083-5093, doi:10.1039/c3ob41007b (2013).

Feula, A., Male, L. \& Fossey, J. S. Diastereoselective preparation of azetidines and pyrrolidines. Org. Lett. 12, 5044-5047, doi:10.1021/ol102215e (2010).

Yoshizawa, A., Feula, A., Leach, A. G., Male, L. \& Fossey, J. S. Palladium and Platinum 2,4-cis-amino Azetidine and Related Complexes. Front Chem.6, doi:10.3389/fchem.2018.00211 (2018).

Yoshizawa, A., Feula, A., Male, L., Leach, A. G. \& Fossey, J. S. Rigid and concave, 2,4-cis-substituted azetidine derivatives: A platform for asymmetric catalysis. Sci. Rep. 8, doi:10.1038/s41598-018-24784-3 (2018).

Birmingham Drug Discovery Facility, <www.birmingham.ac.uk/facilities/bddf> (Date accessed, 15/07/2020). 
The synthesis and preliminary screening data of $\sim 100$ azetidine derivatives that were less active, and not investigated further, will be reported elsewhere.

Palomino, J.-C. et al. Resazurin Microtiter Assay Plate: Simple and Inexpensive Method for Detection of Drug Resistance in Mycobacterium tuberculosis. Antimicrob. Agents Chemother. 46, 2720, doi:10.1128/AAC.46.8.2720-2722.2002 (2002).

Yang, F. et al. Engineering more stable, selectable marker-free autoluminescent mycobacteria by one step. PLoS One 10, e0119341, doi:10.1371/journal.pone.0119341 (2015).

Qin, L. et al. A deletion in the RD105 region confers resistance to multiple drugs in Mycobacterium tuberculosis. BMC Biol. 17, 7, doi:10.1186/s12915-0190628-6 (2019).

Hoagland, D. T., Liu, J., Lee, R. B. \& Lee, R. E. New agents for the treatment of drug-resistant Mycobacterium tuberculosis. Adv Drug Deliv Rev 102, 55-72, doi:10.1016/j.addr.2016.04.026 (2016).

Miles, A. A., Misra, S. S. \& Irwin, J. O. The estimation of the bactericidal power of the blood. Epidemiol. Infect. 38, 732-749, doi:10.1017/S002217240001158X (1938).

Hendon-Dunn, C. L. et al. A Flow Cytometry Method for Rapidly Assessing Mycobacterium tuberculosis Responses to Antibiotics with Different Modes of Action. Antimicrob. Agents Chemother. 60, 3869, doi:10.1128/AAC.02712-15 (2016).

\section{doi:10.1371/journal.pone.0052951 (2012).}

Abrahams, K. A. et al. Identification of KasA as the cellular target of an anti-tubercular scaffold. Nat. Commun. 7, 12581, doi:10.1038/ncomms12581 (2016).

Andries, K. et al. A Diarylquinoline Drug Active on the ATP Synthase of Mycobacterium tuberculosis. Science 307, 223, doi:10.1126/science.1106753 (2005).

Batt, S. M. et al. Whole Cell Target Engagement Identifies Novel Inhibitors of Mycobacterium tuberculosis Decaprenylphosphoryl- $\beta$-d-ribose Oxidase. ACS Infectious Diseases 1, 615-626, doi:10.1021/acsinfecdis.5b00065 (2015).

Manganelli, R., Voskuil, M. I., Schoolnik, G. K. \& Smith, I. The Mycobacterium tuberculosis ECF sigma factor sigmaE: role in global gene expression and survival in macrophages. Mol. Microbiol. 41, 423-437, doi:10.1046/j.1365-2958.2001.02525.x (2001).

Balazsi, G., Heath, A. P., Shi, L. \& Gennaro, M. L. The
Mol. Syst. Biol. 4, 225, doi:10.1038/msb.2008.63 (2008).

Karp, P. D. et al. The BioCyc collection of microbial genomes and metabolic pathways. Brief. Bioinform. 20, 1085-1093, doi:10.1093/bib/bbx085 (2019).

Caspi, R. et al. The MetaCyc database of metabolic pathways and enzymes and the BioCyc collection of pathway/genome databases. Nucleic Acids Res. 44, D471-480, doi:10.1093/nar/gkv1164 (2016).

Chang, Y. \& Fox, B. G. Identification of Rv3230c as the NADPH oxidoreductase of a two-protein DesA3 acyl-CoA desaturase in Mycobacterium tuberculosis H37Rv. Biochemistry 45, 13476-13486, doi:10.1021/bi0615285 (2006).

to redox stress. PLoS One 9, e93604, doi:10.1371/journal.pone.0093604 (2014).
to

Hards, K. et al. Bactericidal mode of action of bedaquiline. J. Antimicrob. Chemother. 70, 2028-2037, doi:10.1093/jac/dkv054 (2015).

Mishra, S. et al. Efficacy of $\beta$-lactam/ $\beta$-lactamase inhibitor combination is linked to WhiB4-mediated changes in redox physiology of Mycobacterium tuberculosis. eLife 6, e25624, doi:10.7554/eLife.25624 (2017).

Waddell, S. J. et al. The use of microarray analysis to determine the gene expression profiles of Mycobacterium tuberculosis in response to anti-bacterial compounds. Tuberculosis 84, 263-274, doi:10.1016/j.tube.2003.12.005 (2004).

Boshoff, H. I. et al. The transcriptional responses of Mycobacterium tuberculosis to inhibitors of metabolism: Novel insights into drug mechanisms of action. J. Biol. Chem. 279, 40174-40184, doi:10.1074/jbc.M406796200 (2004).

Amaral, L. \& Viveiros, M. Thioridazine: A Non-Antibiotic Drug Highly Effective, in Combination with First Line Anti-Tuberculosis Drugs, against Any Form of Antibiotic Resistance of Mycobacterium tuberculosis Due to Its Multi-Mechanisms of Action. Antibiotics 6, doi:10.3390/antibiotics6010003 (2017).

Lee, R. E. et al. Combinatorial lead optimization of [1,2]-diamines based on ethambutol as potential antituberculosis preclinical candidates. J. Comb. Chem. 5, 172-187, doi:10.1021/cc020071p (2003).

Makarov, V. et al. Benzothiazinones Kill Mycobacterium tuberculosis by Blocking Arabinan Synthesis. Science 324, 801, doi:10.1126/science.1171583 (2009).

Xu, Z., Meshcheryakov, V. A., Poce, G. \& Chng, S.-S. MmpL3 is the flippase for mycolic acids in mycobacteria. Proc. Natl. Acad. Sci. U.S.A. 114, 7993, doi:10.1073/pnas.1700062114 (2017).

Favrot, L. et al. Mechanism of inhibition of Mycobacterium tuberculosis antigen 85 by ebselen. Nat. Commun. 4, 2748, doi:10.1038/ncomms3748 (2013).

Ling, L. L. et al. A new antibiotic kills pathogens without detectable resistance. Nature 517, 455-459, doi:10.1038/nature14098 (2015).

Isoniazid was included as a positive control (0.9-29.2 $\mu \mathrm{M})$, and comparison, with it being an antibiotic that targets the cell wall and has rapid bactericidal activity.

Hendon-Dunn, C. L. et al. Regrowth of Mycobacterium tuberculosis Populations Exposed to Antibiotic Combinations Is Due to the Presence of Isoniazid and Not Bacterial Growth Rate. Antimicrob. Agents Chemother. 63, e00570-00519, doi:10.1128/AAC.00570-19 (2019).

Briffotaux, J., Liu, S. \& Gicquel, B. Genome-Wide Transcriptional Responses of Mycobacterium to Antibiotics. Front. Microbiol. 10, doi:10.3389/fmicb.2019.00249 (2019). 
Johnsson, K., King, D. S. \& Schultz, P. G. Studies on the Mechanism of Action of Isoniazid and Ethionamide in the Chemotherapy of Tuberculosis. J. Am. Chem. Soc. 117, 5009-5010, doi:10.1021/ja00122a038 (1995). Milanes, C. L. et al. Altered response of adenylate cyclase to parathyroid hormone during compensatory renal growth. Kidney Int. 36, 802-809,
doi:10.1038/ki.1989.265 (1989). Bacon, J. et al. Non-replicating Mycobacterium tuberculosis elicits a reduced infectivity profile with corresponding modifications to the cell wall and
extracellular matrix. PLoS One 9, e87329, doi:10.1371/journal.pone.0087329 (2014).

Rose, J. D. et al. Synthesis and biological evaluation of trehalose analogs as potential inhibitors of mycobacterial cell wall biosynthesis. Carbohydr. Res. 337, 105-120, doi:10.1016/S0008-6215(01)00288-9 (2002).

Barry, C. S., Backus, K. M., Barry, C. E. \& Davis, B. G. ESI-MS Assay of M. tuberculosis Cell Wall Antigen 85 Enzymes Permits Substrate Profiling and Design of a Mechanism-Based Inhibitor. J. Am. Chem. Soc. 133, 13232-13235, doi:10.1021/ja204249p (2011).

Viljoen, A. et al. Cyclipostins and cyclophostin analogs inhibit the antigen 85C from Mycobacterium tuberculosis both in vitro and in vivo. J. Biol. Chem. 293, 2755-2769 (2018).

Crespo, M., Martinez, M. \& de Pablo, E. Activation volumes for intramolecular oxidative C-X (X = H, F, Cl or Br) addition to platinum(II) imine complexes as a proof of the intimate mechanism. J. Chem. Soc., Dalton Trans., 1231-1236, doi:10.1039/A606872C (1997).

Clark, P. W., Dyke, S. F., Smith, G. \& Kennard, C. H. L. The cyclopalladation of benzylidenebenzylamines. J. Organomet. Chem. 330, 447-460, doi:10.1016/S0022-328X(00)99057-0 (1987). Anderson, C. M., Crespo, M., Kfoury, N., Weinstein, M. A. \& Tanski, J. M. Regioselective C-H Activation Preceded by Csp2-C sp 3 Reductive $_{\text {Elimination from Cyclometalated Platinum(IV) Complexes. Organometallics 32, 4199-4207, doi:10.1021/om400398g (2013). }}$

Zhang, T., Li, S.-Y. \& Nuermberger, E. L. Autoluminescent Mycobacterium tuberculosis for Rapid, Real-Time, Non-Invasive Assessment of Drug and Vaccine Efficacy. PLoS One 7, e29774, doi:10.1371/journal.pone.0029774 (2012).

Liu, Y. et al. The compound TB47 is highly bactericidal against Mycobacterium ulcerans in a Buruli ulcer mouse model. Nat. Commun. 10, 524, doi:10.1038/s41467-019-08464-y (2019).

Nateche, F. et al. Application of the resazurin microtitre assay for detection of multidrug resistance in Mycobacterium tuberculosis in Algiers. J. Med. Microbiol. 55, 857-860, doi:10.1099/jmm.0.46513-0 (2006).

Mosaei, H. et al. Mode of Action of Kanglemycin A, an Ansamycin Natural Product that Is Active against Rifampicin-Resistant Mycobacterium tuberculosis. Mol. Cell 72, 263-274.e265, doi:10.1016/j.molcel.2018.08.028 (2018).

James, B. W., Williams, A. \& Marsh, P. D. The physiology and pathogenicity of Mycobacterium tuberculosis grown under controlled conditions in a defined medium. J. Appl. Microbiol. 88, 669-677, doi:10.1046/j.1365-2672.2000.01020.x (2000). Lambert, R. J. W. Susceptibility testing: inoculum size dependency of inhibition using the Colworth MIC technique. J. Appl. Microbiol. 89, 275-279,
doi:10.1046/j.1365-2672.2000.01105.x (2000).

Lambert, R. J. \& Pearson, J. Susceptibility testing: accurate and reproducible minimum inhibitory concentration (MIC) and non-inhibitory concentration (NIC) values. J. Appl. Microbiol. 88, 784-790, doi:10.1046/j.1365-2672.2000.01017.x (2000).

Luo, S. et al. Effect of HEPES Buffer on the Uptake and Transport of P-Glycoprotein Substrates and Large Neutral Amino Acids. Mol. Pharm. 7, 412420, doi:10.1021/mp900193e (2010).

Borgers, K. et al. Reference genome and comparative genome analysis for the WHO reference strain for Mycobacterium bovis BCG Danish, the present tuberculosis vaccine. BMC Genomics 20, 561, doi:10.1186/s12864-019-5909-5 (2019).

Kapopoulou, A., Lew, J. M. \& Cole, S. T. The MycoBrowser portal: A comprehensive and manually annotated resource for mycobacterial genomes. Tuberculosis 91, 8-13, doi:10.1016/j.tube.2010.09.006 (2011).

Cox, J. A. G. et al. THPP target assignment reveals EchA6 as an essential fatty acid shuttle in mycobacteria. Nat. Microbiol. 1, 15006, doi:10.1038/nmicrobiol.2015.6 (2016).

Abrahams, K. A. et al. Inhibiting mycobacterial tryptophan synthase by targeting the inter-subunit interface. Sci. Rep. 7, 9430, doi:10.1038/s41598-01709642-y (2017).

Briffotaux, J., Huang, W., Wang, X. \& Gicquel, B. MmpS5/MmpL5 as an efflux pump in Mycobacterium species. Tuberculosis 107, 13-19, doi:10.1016/j.tube.2017.08.001 (2017).

Abrahams, K. A. et al. Identification of Novel Imidazo[1,2-a]pyridine Inhibitors Targeting M. tuberculosis QcrB. PLoS One 7, e52951, doi:10.1371/journal.pone.0052951 (2013). 